\title{
On the Scalar Rational Interpolation Problem
}

\author{
A. C. Antoulas $\dagger$ \\ Department of Electrical and Computer Engineering, Rice University, Houston, \\ Texas 77251-1892, U.S.A.
}

AND

Mathematical System Theory, E.T.H. Zürich, CH-8092 Zürich, Switzerland

AND

B. D. Q. ANDERSON

Department of Systems Engineering, Australian National University, Canberra, A.C.T. 2601, Australia

[Received 22 November 1985 and in revised form 29 April 1986]

The rational interpolation problem in the scalar case, including multiple points, is solved. In particular a parametrization of all minimal-degree rational functions interpolating given pairs of points is derived. These considerations provide a generalization of the results on the partial realization of linear systems.

\section{Introduction}

CONSIDER the pairs of points $\left(x_{i}, y_{i}\right)(i=1, \ldots, N)$, where each entry belongs to some arbitrary but fixed infinite field. The fundamental problem to be investigated is to parametrize all rational functions

$$
y(x)=n(x) / d(x),
$$

in particular the ones having minimal complexity, which interpolate the above points. If these points are distinct, i.e. $x_{i} \neq x_{j}$ for $i \neq j$, then we must have $y\left(x_{i}\right)=y_{i}(i=1, \ldots, N)$.

The straightforward approach to the problem is the following. Let $y(x)$, defined by (1.1), be a rational function of degree $m$, i.e.

$$
\operatorname{deg} y:=\max \{\operatorname{deg} n, \operatorname{deg} d\}=m \text {. }
$$

We define $X$ to be the $N \times(m+1)$ Vandermonde matrix whose ith row is $\boldsymbol{x}_{i}^{\top}=\left[1, x_{i}, \ldots, x_{i}^{m}\right]$, and $Y:=\operatorname{diag}\left(y_{1}, \ldots, y_{N}\right)$ (it is assumed for simplicity that all pairs $\left(x_{i}, y_{i}\right)$ are finite and distinct). Let $v$ and $\delta$ be $(m+1) \times 1$ column vectors containing the coefficients of the polynomials $n(x)$ and $d(x)$, starting with

† Most of this research was conducted while the first author was a Visiting Fellow at the Australian National University, August-September 1985. Support was also provided by N.S.F. through Grant ECS-8505293. 
the constant term. It is readily checked that one parametrization of the set of all interpolating functions of degree at most $m$, is given as follows:

$$
[X-Y X]\left[\begin{array}{l}
v \\
\delta
\end{array}\right]=0
$$

subject to the constraints

$$
\boldsymbol{x}_{i}^{\top} \boldsymbol{\delta} \neq 0 \quad(i=1, \ldots, N)
$$

which ensure that $x_{i}(i=1, \ldots, N)$ is not a common factor of the polynomials $n(x)$ and $d(x)$. The problem thus reduces to finding those $m$ for which equations (1.2), subject to (1.3), have a solution. The difficulty with this setting is that $m$ is not properly encoded in $X$ and $Y$, and can be deduced only by trial and error. The need for a different approach, i.e. a repackaging of the data, becomes apparent.

For this purpose, we notice that one rational interpolating function $y(x)$ is determined by:

$$
\sum_{i=1}^{N} c_{i} \frac{y(x)-y_{i}}{x-x_{i}}=0, \quad c_{i} \neq 0 .
$$

Clearly $y\left(x_{i}\right)=y_{i}$ if $c_{i} \neq 0$. Depending on the particular choice of the $c_{i}$ 's, the degree of $y(x)$ is at most $N-1$ (generically this upper bound is attained).

As already mentioned, our goal is to investigate the algebraic structure of the problem of parametrizing all interpolating functions, in particular those of minimal complexity (degree). One way for doing this is to try to determine those non-zero values of the coefficients $c_{i}(i=1, \ldots, N)$ in (1.4) for which we have the greatest number of pole-zero cancellations between the numerator and denominator polynomials of $y$. Another way for minimizing the degree of $y$, which is the one we have adopted, is the following. We consider a summation as in (1.4) containing only $q<N$ summands; for any set of non-zero $c_{i}$, the rational function $y$, of generic degree $q-1$, interpolates the first $q$ points. Making use of the freedom in choosing the $c_{i}$, we then try to achieve the interpolation of the $N-q$ points. Let

$$
c:=\left[\begin{array}{lll}
c_{1} & \cdots & c_{q}
\end{array}\right]^{\mathrm{T}}
$$

in order for the remaining $N-q$ points to be interpolated, $c$ must be in the kernel of the $(N-q) \times q$ matrix

$$
L:=\left[\frac{y_{i}-y_{l}}{x_{i}-x_{j}}: j=1, \ldots, q ; i=q+1, \ldots, N\right] .
$$

This is a Löwner or divided-differences matrix derived from the given (distinct) pairs of points. The corresponding matrix for multiple points is called generalized Löwner matrix. The (generalized) Löwner matrix turns out to be the fundamental tool for the investigation of the rational interpolation problem. The main property of this matrix is that its rank is related in a simple way, to the degree of the corresponding minimal-degree interpolating function(s). 
The main result of Section 2 asserts that the minimal degree of the interpolating function(s) is either rank $L$ or $N-\operatorname{rank} L$, according to whether certain explicitly stated conditions are satisfied or not. In the former case the minimal interpolating function is unique, while in the latter it is nonunique, having $N-2$ rank $L+1$ degrees of freedom. There follows a parametrization of all minimal and nonminimal interpolating functions in the form (1.4), for appropriate $q$ and $c$. The third section deals with the problem of recursiveness. The main question is how to update (minimally) the interpolating function whenever additional points are provided, without having to start from scratch. It is first shown how to parametrize all minimal interpolating functions, given a single one of them; the second step consists in showing how to determine one minimal updating of a given interpolating function. These two results combined provide a parametrization of all minimal updatings. The investigation of recursiveness is based on a linear fractional representation formula, much as in the partial realization case (see Antoulas (1985)). The results just described have been derived for the general case of multiple interpolation points.

The (partial) realization problem of linear system theory, can be viewed as a special case of the rational interpolation problem, where all the $x_{i}$ 's are the same (conventionally taken to be the point at infinity). The main tool for the study of the (partial) realization problem is the (partially defined) Hankel matrix (see e.g. Kalman (1979) and Bosgra (1983)). The question arises as to what the generalization of the Hankel matrix is in the case of the general interpolation problem. An important consequence of our approach is the fact that the generalized Löwner matrix, defined for pairs of points with the same $x_{i}$ 's, has Hankel structure, and indeed is part of the Hankel matrix of the corresponding partial realization problem. This shows that in the context of interpolation problems, Hankel matrices are generalized to Löwner matrices. Thus the theory of the (scalar) rational interpolation problem presented in this paper constitutes the generalization of the (scalar partial) realization problem.

The interpolation problem has numerous applications in network, system and control theory. A classical paper on the use of interpolation in network and system theory is Youla \& Saito (1967). More recent references include Chang \& Pearson (1984), Anderson \& Linnemann (1985), to mention only two. In the first, the close connection between $H^{\infty}$-optimization in linear control systems and the interpolation problem (with stability requirements) is demonstrated. In the second it is shown that a problem of compensator complexity in decentralized control reduces to an interpolation problem.

\section{The minimal-interpolation problem}

Consider the array of point pairs

$$
P:=\left(\left(x_{i}, y_{i, j-1}\right): j=1, \ldots, v_{i}, i=1, \ldots, \theta\right),
$$

consisting of $N:=v_{1}+\cdots+v_{\theta}$ pairs; $v_{i}$ is the multiplicity of $x_{i}$; the array $P$ is said to contain distinct pairs if $v_{l}=1$, for all $i$ (for simplicity of notation, in this case $y_{i}:=y_{10}$ ). We will assume in the sequel that the $x$ 's and the $y$ 's are finite (see 
Remark 2.30a). A rational function $y(x)$ is said to interpolate $\left(x_{i}, y_{i, j-1}\right)$ iff $\mathrm{D}^{\prime-1} y\left(x_{i}\right)=y_{i, j-1} \quad\left(j=1, \ldots, v_{i}\right)$, where $\mathrm{D}$ denotes derivation with respect to $x$. Let $Q$ denote the array containing the $x_{i}$, where each one is listed $v_{i}$ times; $Q=\left(x_{1}, \ldots, x_{1} ; \ldots ; x_{\theta}, \ldots, x_{\theta}\right)$; thus $Q$ contains $N$ elements.

We partition $Q$ in two disjoint arrays $S$ and $T$ called the row array and the column array, respectively, with: $S=\left(s_{1}, \ldots, s_{r}\right)$ and $T=\left(t_{1}, \ldots, t_{N-r}\right)$ such that

$$
s_{i}, t_{i} \in\left\{x_{1}, \ldots, x_{\theta}\right\}, \quad \#\left\{k: s_{k}=x_{i} \text { and } t_{k}=x_{i}\right\}=v_{i} \text {. }
$$

We denote by $i^{\prime}$ and $j^{\prime}$ the indices such that $s_{i}=x_{i}$, and $t_{j}=x_{i \prime}$, respectively.

To each such partitioning of $Q$, we associate an $r \times(N-r)$ matrix denoted by $L$ and referred to as Löwner or generalized Löwner matrix, according to whether $v_{i}=1$ for all $i$, or $v_{i}>1$ for some $i$. The $(i, j)$ th element of $L$ is defined as

$$
\ell_{i j}:=\frac{y\left(s_{i}\right)-y\left(t_{j}\right)}{s_{i}-t_{j}} \quad(i=1, \ldots, r ; j=1, \ldots, N-r),
$$

where $y\left(s_{i}\right)=y_{i}$, and $y\left(t_{j}\right)=y_{j^{\prime}}$, provided all pairs are distinct. In case of multiple points, we assume that they have consecutive indices in both $S$ and $T$. Let, for example,

$$
\begin{aligned}
s_{i} & =s_{i-1}=\cdots=s_{i-k} \neq s_{i-m} \quad(m>k), \\
t_{j} & =t_{j-1}=\cdots=t_{j-l} \neq t_{j-m} \quad(m>l) .
\end{aligned}
$$

The $(i, j)$-th element of $L$ in this case is defined as follows, if $s_{i} \neq t_{j}$ :

$$
\ell_{i j}:=\mathrm{D}_{s}^{k} \mathrm{D}_{t}^{\prime}\left[\frac{y(s)-y(t)}{s-t}\right]_{s-s_{b}, t=t_{j}} \quad(i=1, \ldots, r ; j=1, \ldots, N-r)
$$

where $D_{s}^{k}\left(D_{t}^{\prime}\right)$ denote the $k$ th derivative with respect to $s$ (lth derivative with respect to $t$ ) and

$$
\mathrm{D}_{s}^{p-1} y\left(s_{i}\right):=y_{i^{\prime}, p-1}, \quad \mathrm{D}_{i}^{q-1} y\left(t_{i}\right):=y_{j^{\prime}, q-1} ;
$$

if $s_{i}=t_{j}=t$ we have to compute the limit of the above expression as $s_{i}$ tends to $t_{j}$ (clearly $i^{\prime}=j^{\prime}$ ). A straightforward computation using the Taylor expansion of $y(s)$ in the neighbourhood of $s=t$, gives

$$
\ell_{i j}=\frac{k ! l !}{(k+l+1) !} \mathrm{D}_{t}^{(k+l+1)} y(t)=\frac{k ! l !}{(k+l+1) !} y_{l^{\prime}, k+l+1} \text {. }
$$

2.4 ExAMPLE Let $P$ contain 6 pairs of points: $\left(x_{1}, y_{1}\right),\left(x_{2}, y_{20}\right),\left(x_{2}, y_{21}\right)$, $\left(x_{2}, y_{22}\right),\left(x_{2}, y_{23}\right),\left(x_{3}, y_{3}\right)$. We will compute the generalized Lowner matrix corresponding to the following partitioning of the $x$ 's: $s_{1}=x_{3}, s_{2}=s_{3}=x_{2}$, i.e. $S=\left(x_{3}, x_{2}, x_{2}\right) ; t_{1}=x_{1}, t_{2}=t_{3}=x_{2}$, i.e. $T=\left(x_{1}, x_{2}, x_{2}\right)$. The resulting $L$ is:

$$
L=\left[\begin{array}{ccc}
\frac{y_{3}-y_{1}}{x_{3}-x_{1}} & \frac{y_{3}-y_{20}}{x_{3}-x_{2}} & \frac{y_{3}-y_{20}}{\left(x_{3}-x_{2}\right)^{2}}-\frac{y_{21}}{x_{3}-x_{2}} \\
\frac{y_{20}-y_{1}}{x_{2}-x_{1}} & \frac{y_{21}}{1 !} & \frac{y_{22}}{2 !} \\
-\frac{y_{20}-y_{1}}{\left(x_{2}-x_{1}\right)^{2}}+\frac{y_{21}}{x_{2}-x_{1}} & \frac{y_{22}}{2 !} & \frac{y_{23}}{3 !}
\end{array}\right] .
$$


Of fundamental importance is the equality of the degree of a rational function and the rank of an associated Löwner (or generalized Löwner) matrix of large enough size. For a different proof of this result in the case of distinct points, see Belevitch (1970).

2.5 MAIN LEMMA Consider the rational function $y(x)$. Let $L$ be any $\sigma \times \tau$ (generalized) Löwner matrix corresponding to the $\sigma+\tau$ (not necessarily distinct) points whose $x$-values are $s_{i}$ and $t_{j}(i=1, \ldots, \sigma ; j=1, \ldots, \tau)$. Then $L$ has the property:

$$
\text { rank } L=\operatorname{deg} y=: q, \quad \sigma, \tau \geqslant \operatorname{deg} y,
$$

where $L$ is defined by $(2.3 \mathrm{a}, \mathrm{b})$.

In the sequel we will also make use of the $(\sigma+1) \times(\tau-1)$ Löwner matrix denoted by $L^{*}$, which is obtained from $L$ by deleting a single occurrence of $t_{j}$ from $T$, to form an array $T^{*}$, and adjoining it to $S$ to form an array $S^{*}$.

2.6 COROLLARY Under the assumptions of the lemma, all $q \times q$ (generalized) Löwner submatrices of $L$ and $L^{*}$ are nonsingular.

2.7 Remark. Any submatrix of a Löwner matrix is also a Löwner matrix. This is not true however with generalized Löwner matrices. For this to happen, if the submatrix in question contains the $(i, j)$ th element of $L$, it should also contain the elements: $(i, j-m)(m=1, \ldots, l)$ and $(i-m, j)(m=1, \ldots, k)$ of $L$ where (2.2) is assumed to hold.

Proof of Lemma 2.5 (Sketch). Let $y(x)=n(x) / d(x)$, where $n$ and $d$ are coprime, and $\operatorname{deg} y=q$. We denote by $B$ the $q \times q$ Bezoutian of the polynomials $n$ and $d$; it is well known that $B$ is non-singular if and only if the polynomials $n$ and $d$ are coprime. For the definition and properties of the Bezoutian of two polynomials, see Fiedler (1984), as well as Anderson \& Jury (1976). The following holds

$$
v^{\top} B w=\frac{n(s) d(t)-n(t) d(s)}{s-t}=d(s) \frac{y(s)-y(t)}{(s-t)} d(t)
$$

where

$$
\boldsymbol{v}^{\top}:=\left[1, s, \cdots, s^{q-1}\right], \quad \boldsymbol{w}^{\top}:=\left[1, t, \cdots, t^{q-1}\right] .
$$

Let $S:=\left(s_{i}: i=1, \ldots, \sigma\right)$ and $T:=\left(t_{j}: j=1, \ldots, \tau\right)$ be given. Formula (2.8a) implies

$$
V B W=\Delta, L \Delta_{t}
$$

where $V$ is the $\sigma \times q$ Vandermonde matrix whose $i$ th row is $\left[1, s_{i}, \cdots, s^{q^{-1}}\right]$, while $W$ is the $q \times \tau$ Vandermonde matrix whose $j$ th column is $\left(1, t_{j}, \cdots, t q^{-1}\right)$,

$$
\Delta_{s}:=\operatorname{diag}\left[d\left(s_{1}\right), \ldots, d\left(s_{\sigma}\right)\right], \quad \Delta_{t}:=\operatorname{diag}\left[d\left(t_{1}\right), \ldots, d\left(t_{\tau}\right)\right],
$$

and

$$
\ell_{i j}=\frac{y\left(s_{i}\right)-y\left(t_{j}\right)}{s_{i}-t_{j}}
$$


We assume that none of the $s_{i}$ or $t_{j}$ is a pole of $y(x)$. If these points are distinct then $L$ is the Lowner matrix constructed with row and column arrays $S$ and $T$ (cf. (2.1)). In this case we can rewrite

$$
L=\Delta_{s}^{-1} V B W \Delta_{t}^{-1} .
$$

Since $n$ and $d$ are coprime, $B$ is nonsingular. Thus if $\sigma$ and $\tau$ are greater than $q$,

$$
\operatorname{rank} L=\operatorname{rank} B=q
$$

If the entries in $S$ and $T$ are not distinct, $L$ has to be replaced by the generalized Löwner matrix, denoted by $\bar{L}$ (cf. $(2.3 \mathrm{a}, \mathrm{b})$ ); in the remaining part of the paper after the end of this proof, for simplicity of notation, the bar will be dropped.

Let $S$ have one multiple entry $s_{1}$, of multiplicity $v$, i.e., $s_{1}=s_{2}=\cdots=s_{v}$. In this case, the Vandermonde matrix $V$ does not have full rank, and thus the ranks of $L$ and $B$ are not the same. In order to find a matrix $\bar{L}$ whose rank is equal to that of $B$, we proceed as follows. Assuming temporarily, that $s_{1}, \ldots, s_{v}$ are different, we differentiate the expression in (2.8c) $i$ times with respect to $s_{i+1}(i=1, \ldots, v-1)$, and subsequently set $s_{2}, \ldots, s_{v}$ equal to $s_{1}$; let us denote by $\bar{D}:=D_{s_{v}}^{v-1} \cdots D_{s_{3}}^{2} D_{s_{2}}^{1}$, these successive derivations. Then

$$
\bar{D} L=D\left(\Delta_{s}^{-1} V B W \Delta_{t}^{-1}\right)=D\left(\Delta_{s}^{-1} V\right) B W \Delta_{t}^{-1} \text {. }
$$

It is readily checked that

$$
\bar{D}\left(\Delta_{s}^{-1} V\right)=E^{\top} \bar{D} V
$$

where $e_{i+1}^{\top}=\left[\gamma_{i l} \delta^{(l)}, \gamma_{l, i-1} \delta^{(i-1)}, \ldots, \gamma_{i 0} \delta, 0, \ldots, 0\right]$ is the $(i+1)$ th row of $E$,

$$
\gamma_{i j}:=\frac{i !}{(i-j) ! j !} \quad(j \leqslant i)
$$

is the corresponding coefficient of the binomial expansion, and $\delta^{(j)}$ denotes the $j$ th derivative of $d^{-1}\left(s_{i}\right)$ with respect to $s_{i}$. Thus

$$
\bar{L}:=[D L]_{s_{2}=\cdots s_{s}=s_{1}}=\left[E(D V) B W \Delta_{t}^{-1}\right]_{s_{2}-\cdots=s_{v}=s_{1}}
$$

has the same rank as $B$, since $E$ is nonsingular, and $[D V]_{s_{2}=\cdots s_{v}=s_{1}}$ is the corresponding generalized Vandermonde matrix which has full rank (see e.g. Aitken (1964)). $\bar{L}$ as constructed above is the generalized Löwner matrix defined in (2.3a).

In a similar way, more than one multiple point in $S$ and multiple point in $T$ can be treated. If in addition some points in $S$ are equal to some points in $T$, the resulting expression is the one given in (2.3b).

Proof of Corollary 2.6. The result is an immediate consequence of the lemma. The restriction to generalized Löwner submatrices (see Remark 2.7) follows from the nature of the $\bar{D}$ operator, defined in the proof.

To every $r \times(N-r)$ Löwner matrix satisfying rank $L<N-r$, one can attach a rational function as follows. Let $c=\left[c_{1}, \ldots, c_{N-r}\right]^{\top} \neq 0$ be such that $L c=0$. A 
rational function $y_{L}(x)$ is defined through the equation:

$$
\sum_{j=1}^{N-r} c_{j} \frac{y_{L}(x)-y\left(t_{j}\right)}{x-t_{j}}=0
$$

Similarly, if rank $L<r$, a row vector $\boldsymbol{b}^{\top}=\left[b_{1}, \ldots, b_{r}\right] \neq \mathbf{0}$, satisfying $\boldsymbol{b}^{\top} L=\mathbf{0}$, associates a rational function to $L$ as well.

To every generalized Löwner matrix one can also attach a rational function. Again let $c$ be a non-zero column vector such that $L c=0$. With

$$
p_{L}(x, t):=\frac{y_{L}(x)-y(t)}{x-t},
$$

a rational function $y_{L}(x)$ can be defined as follows.

$$
\left[\sum_{i, j} c_{i, j-1} \mathrm{D}_{t}^{-1} p_{L}(x, t)\right]_{t=t_{i}}=0,
$$

where $j$ ranges from 1 to the multiplicity $v_{i}^{\prime}$ of $t_{i}$ in $T$, and $t_{i}$ ranges over the $\theta^{\prime}$ distinct points of $T$; also, the $j$ th derivative of $y(t)$ with respect to $t$, evaluated at $t=t_{i}$ is $y_{i^{\prime} ;}$; finally

$$
c_{i j} \text { is the }\left(v_{1}^{\prime}+v_{2}^{\prime}+\cdots+v_{i-1}^{\prime}+j+1\right) \text { th element of } c \text {. }
$$

A similar construction can be carried out, based on a non-zero row vector $b^{\top}$ satisfying $\boldsymbol{b}^{\top} L=0$.

Solving (2.10) with respect to $y_{L}(x, c)$ (with the dependence on $c$ shown explicitly) we obtain

$$
y_{L}(x, c)=n_{L}(x, c) / d_{L}(x, c) ;
$$

the numerator and denominator polynomials are defined as follows:

$$
n_{L}(x, c):=p^{\top}(x) Y c, \quad d_{L}(x, c):=p^{\top}(x) c ;
$$

$p^{\top}(x)$ is a row vector of size $N-r$ :

$$
\begin{gathered}
p^{\top}(x):=\left[\begin{array}{lll}
p_{1}(x) & \cdots & p_{\theta^{\prime}}(x)
\end{array}\right] \\
\left(v_{i}^{\prime}-1\right) ! p_{i}(x):=\left[\begin{array}{llll}
\left(x-x_{i}\right)^{v_{i}-1} & \cdots & \left(x-x_{i}\right) & 1
\end{array}\right] \prod_{j \neq i}\left(x-x_{j}\right)^{v_{i}}
\end{gathered}
$$

$x_{j}$ ranges over the $\theta^{\prime}$ distinct entries of $T$. Also,

$$
Y:=\operatorname{diag}\left(Y_{1}, \ldots, Y_{\theta^{\prime}}\right) \text {, }
$$

where each $Y_{i}$ is an upper-triangular square Töplitz matrix of size $v_{l}^{\prime}$, with the $(j+1)$ th element of the first row equal to $y_{i j} / j !\left(j=0,1, \ldots, v_{i}^{\prime}-1\right)$. Finally $c=\left[c_{1}, \ldots, c_{\theta^{\prime}}\right]^{\top}$ is a column vector of size $N-r$ where $\theta^{\prime}$ is the number of distinct $x_{i}$ 's in the array $T$; each component of $c$ is

$$
c_{i}:=\left[c_{i 0}, c_{i 1}, \ldots, c_{i, v_{i}^{\prime}-1}\right]^{\top}
$$

where $v_{i}^{\prime}$ is the multiplicity of $x_{l}$ in the array $T$. In the above considerations we have assumed that equal $x$ 's have consecutive indices. 
Notice that the degree of $y_{L}$ constructed above, is at most $N-r-1$. For future use we note that the coefficient of the highest power of $x$ in the denominator of $y_{L}$ is:

$$
c_{10}+c_{20}+\cdots+c_{\theta^{\prime} 0}
$$

2.13 Proposition The pair of polynomials $n_{L}$ and $d_{L}$ given by (2.11a-e), satisfies for each multiple point $\left(x_{i}, y_{i, j-1}\right)\left(j=1, \ldots, v_{l}\right)$ the following system of linear equations:

$$
A_{i} y_{i}^{*}=b_{i} \quad(i=1, \ldots, \theta) .
$$

Here, $A_{i}$ is a square, lower-triangular matrix of size $v_{i}$; its $(k, l)$ th element is

$$
\gamma_{k-1, l-1} \mathrm{D}^{k-l} d_{L}\left(x_{i}, c\right) \quad\left(l=1, \ldots, k ; k=1, \ldots, v_{i}\right),
$$

with the $\gamma^{\prime}$ 's as defined previously; $\boldsymbol{y}_{i}^{*}:=\left[y_{i 0}, y_{i 1}, \ldots, y_{i, v_{i}-1}\right]^{\top} ; b_{i}$ is a column vector of size $v_{i}$; its $k$ th element is $D^{k-1} n_{L}\left(x_{i}, c\right)\left(k=1, \ldots, v_{i}\right)$; D denotes derivation with respect to $x$.

The proof of this proposition involves straightforward but rather tedious algebraic manipulations and will be omitted. We just mention that for points belonging to the column array $T$, the corresponding number of equations in (2.14) are satisfied for all values of $c$, while for the remaining points, the fact that $c$ is in the kernel of $L$ has to be used.

2.15 COROLlaRY The rational function $y_{L}$ interpolates the multiple point $\left(x_{i}, y_{i, j-1}\right)$ in $P$, if $d_{L}\left(x_{i}, c\right) \neq 0$, i.e. if $x-x_{i}$ is not a common factor of $n_{L}$ and $d_{L}$, given by (2.11).

Proof. From (2.14) follows that $y_{L}$ interpolates each multiple point, provided that $A_{t}$ is non-singular. Since $A_{i}$ is triangular with $d_{L}\left(x_{i}, c\right)$ on the diagonal, the desired conclusion follows.

2.16 Examples. (a) In Example 2.4, suppose that there exists $c=$ $\left[c_{10}, c_{20}, c_{21}\right]^{\top} \neq 0$ such that $L c=0$. The rational function $y_{L}$ attached to $L$ is: $y_{L}(x, c)=n_{L}(x, c) / d_{L}(x, c)$, where

$$
\begin{aligned}
& n_{L}(x, c)=\tilde{c}_{10}\left(x-x_{2}\right)^{2}+\tilde{c}_{20}\left(x-x_{1}\right)\left(x-x_{2}\right)+\bar{c}_{21}\left(x-x_{1}\right), \\
& d_{L}(x, c)=c_{10}\left(x-x_{2}\right)^{2}+c_{20}\left(x-x_{1}\right)\left(x-x_{2}\right)+c_{21}\left(x-x_{1}\right),
\end{aligned}
$$

and

$$
\bar{c}_{10}=c_{10} y_{10}, \quad \bar{c}_{20}=c_{20} y_{20}+c_{21} y_{21}, \quad \bar{c}_{21}=c_{21} y_{20} .
$$

(b) If $v_{i}^{\prime}=1\left(i=1, \ldots, \theta^{\prime}\right)$, then

$$
n_{L}(x, c)=\sum_{i=1}^{\theta^{\prime}} c_{i 0} y_{i 0} \prod_{j \neq i}\left(x-x_{j}\right), \quad d_{L}(x, c)=\sum_{i=1}^{\theta^{\prime}} c_{i 0} \prod_{j \neq i}\left(x-x_{j}\right) .
$$

We now turn our attention to the investigation of the basic properties of $y_{L}$, defined by (2.11), where $L$ is square or almost square, i.e. the difference of the number of rows and the number of columns is 0 or \pm 1 . In the remainder of this section we will use the notation

$$
m:=\frac{1}{2} N \text {, if } N \text { is even, and } m:=\frac{1}{2}(N-1) \text {, if } N \text { is odd. }
$$


The first result shows that to every square or almost square Löwner matrix with nonzero (column or row) kernel, formulae (2.11) associate a unique rational function (see also Remark 2.20b).

2.17 LEMMA Let $L$ be some $m \times m$ or $m \times(m+1)$ Löwner matrix formed from the $N$ pairs of points in $P$, with rank $L \leqslant m$, where equality holds only if $N$ is odd. There exists a unique rational function attached to $L$ via (2.11).

Proof. Assume, for simplicity, that $N$ is even (similar arguments hold for $N$ odd). There exists a column vector $c$ such that $L c=0$. Let $b$ be a column vector satisfying either $b^{\top} L=0$ or $L b=0$, with $b \neq c$. Suppose that $y_{c}=n_{c} / d_{c}$ and $y_{b}=n_{b} / d_{b}$ are rational functions of degrees $q_{c}$ and $q_{b}$ constructed using formulae (2.11). The degree of both $y_{c}$ and $y_{b}$ is at most $m-1$. Thus, by Corollary $2.15 y_{c}$ interpolates at least $N-$ (number of common factors between $d_{c}$ and $\left.n_{c}\right)=$ $N-\left(m-1-q_{c}\right)=N-m+1+q_{c}$ points of $P$ and, similarly $y_{b}$, interpolates at least $N-m+1+q_{b}$ points. It follows that $y_{c}$ and $y_{b}$ interpolate at least

$$
N-2 m+q_{c}+q_{b}+2=q_{c}+q_{b}+2>q_{c}+q_{b}
$$

points in common among the $N$ given. This implies

$$
y_{c}(x)-y_{b}(x)=r(x) \prod_{i}\left(x-x_{i}\right)
$$

where $x_{i}$ are the common interpolation points and $r(x)$ is some rational function with poles different from the $x_{i}$ 's. The rational function on the left has degree at most $q_{c}+q_{b}$, while the one on the right has degree at least $q_{c}+q_{b}+2$. Thus equality can hold only if $r(x)=0$, which implies $y_{c}=y_{b}$.

The converse of Corollary 2.15 is given next.

2.18 Corollary Let $L$ be as in the lemma. If $\left(x-x_{i}\right)^{\alpha_{i}}(i=1, \ldots, \theta)$, is a common factor of $n_{L}$ and $d_{L}$ defined by (2.11), $y_{L}$ interpolates exactly $\left(x_{i}, y_{i, j-1}\right)$, for $j=1, \ldots, v_{i}-\alpha_{i}$ and $i=1, \ldots, \theta$.

Proof. Let $d_{L}=\left(x-x_{i}\right)^{\alpha_{i}} \bar{d}_{L}$, and $n_{L}=\left(x-x_{i}\right)^{\alpha_{i}} \bar{n}_{L}$, with $\alpha_{i} \leqslant v_{i}$. Substituting these expressions in (2.14), the first $\alpha_{l}$ equations turn out to be of the form $0=0$. The remaining $v_{l}-\alpha_{i}$, can be written in matrix form as $\bar{A}_{i} \bar{y}_{i}=\bar{b}_{i}$, where the matrix $\bar{A}_{i}$, and the column vectors $\tilde{y}_{i}^{*}$ and $\bar{b}_{i}$, are defined the same way as their unbarred counterparts in (2.14), with $v_{i}$ replaced by $v_{i}-\alpha_{i}, n_{L}$ by $\bar{n}_{L}$, and $d_{L}$ by $\bar{d}_{L}$. Thus, $y_{L}=\bar{n}_{L} / \bar{d}_{L}$ interpolates $\left(x_{i}, y_{i, j-1}\right)$, for $j=1, \ldots, v_{i}-\alpha_{i}$ and $i=$ $1, \ldots, \theta$.

To prove that $y_{L}$ does not interpolate any of the remaining points of $P$ we proceed as follows.

By Lemma 2.17, $y_{L}$ is independent of the choice of $c$. Let rank $L=q$; assume for simplicity, that the first $q$ columns of $L$ are linearly independent. Then, $c$ satisfying $L c=0$, can be chosen as follows:

$$
c=\left[\begin{array}{llllll}
c_{1} & \cdots & c_{q+1} & 0 & \cdots & 0
\end{array}\right]^{\top}
$$

Thus, the degree of $y_{L}$ is at most $q$. Let the degree of the greatest common 
divisor of $n_{L}$ and $d_{L}$ be $\mu$; then $\operatorname{deg} y_{L}=q-\mu$. Assume that $y_{L}$ interpolates more than $N-\mu$ points, namely $N-\mu+\pi$, with $\pi \geqslant 0$. We will show that $\pi=0$.

Let $L_{1}$ be the Löwner matrix obtained from $L$ by deleting the rows and the columns which correspond to the $\mu-\pi$ points which are not interpolated by $y_{L}$; we have rank $L_{1} \geqslant q-\mu+\pi$. By construction, however, all the points making up $L_{1}$ are interpolated by $y_{L}$, which has degree $q-\mu$. Main Lemma 2.5 implies that the rank of $L_{1}$ is equal to $q-\mu$, which in turn implies $\pi=0$.

From Corollary 2.18 we obtain immediately the following crucial result.

2.19 COROLLARY Under the assumptions of the lemma, let $\bar{L}$ be an arbitrary full rank $q \times(q+1)$ Löwner submatrix of $L$, where $q:=\operatorname{rank} L$. The following statements are equivalent.

(a) $y_{L}$ interpolates all pairs of points in $P$.

(b) All $q \times q$ Löwner submatrices of $\bar{L}$ and $\bar{L}^{*}$ are nonsingular.

(c) $\operatorname{deg} y_{L}=\operatorname{rank} L=q$.

(d) $x_{i}$ is not a common root of $n_{L}$ and $d_{L}$, for all $i=1, \ldots, \theta$.

Proof. By Corollary (2.6), (a) implies (b).

Let $\bar{c}$ be such that $\bar{L} \bar{c}=0$. According to (2.17) there exists a unique rational function $y_{L}$, attached to $\bar{L}$. (b) implies $\operatorname{deg} y_{L}=q$. There exists a column vector $\boldsymbol{c} \neq \mathbf{0}$ composed of the elements of $\bar{c}$ and of zeros in appropriate positions, such that $L c=0$. Since by (2.17) there is a unique rational function $y_{L}$ attached to $L$, we have $y_{L}=y_{L}$. This implies (c).

Since the degree of $y_{L}$ is at most $q$, (c) implies (d).

Finally, by (2.15), (d) implies (a).

2.20 Remarks. (a). From the arguments used in the proof of Lemma 2.17, it follows that $y_{L}$ interpolates at least $N-m+\operatorname{deg} y_{L}$ points. Considering Corollary 2.19(c), we conclude that $y_{L}$ interpolates exactly $N-q+\operatorname{deg} y_{L}$ points.

(b). The considerations of Lemma 2.17 and Corollary 2.19 remain valid if, instead of being (almost) square, $L$ is taken to be some Lowner matrix having rank $q$ (see Corollary 2.24). If however, with the same points, a Löwner matrix $L^{\prime}$ of size $r \times(N-r)$, with $r<q$, is formed, then the rank of $L^{\prime}$ will be (at most) $r$. In this case Lemma 2.17 does not apply. Actually, a number of different rational functions are associated to $L^{\prime}$ via $(2.5,6)$ (see $(2.26)$, and Remark $2.30 \mathrm{c}$ ).

(c). By Corollary 2.19, if there exists one Löwner submatrix $\bar{L}$ of $L$ which does not satisfy the conditions stated in Corollary $2.19(\mathrm{~b})$, there exists none which does so.

Corollary 2.19 implies therefore, that in our study of the interpolation problem, we can restrict our attention to any arbitrary $q \times(q+1)$ full-rank Löwner submatrix $\bar{L}$ of $L$, where $q$ is the rank of $L$.

(d). Let $\bar{L}$ as in Corollary 2.19 have row and column sets $\bar{S}$ and $\bar{T}$. For $x_{i} \in \bar{T}$, Corollary 2.19 (d) reduces to $c_{i, v_{i}-1} \neq 0$, where $v_{i}^{\prime}$ is the multiplicity of $x_{i}$ in $\bar{T}$.

If the conditions of Corollary 2.19 are not satisfied, the theorem below shows that the functions interpolating the given $N$ pairs of points $P$, have least degree $N-q$. 
2.21 THEOREM Consider the array of $N$ pairs of points $P$, and some associated $m \times m$ or $m \times(m+1)$ (generalized) Löwner matrix L. Assume that $\operatorname{deg} y_{L}<$ rank $L:=q$. There exists a rational function of degree $N-q$ interpolating all the points in $P$. Furthermore, no such function of degree less than $N-q$ exists.

The proof of this theorem is based on the following lemma.

2.22 LEMMA (Extension of Löwner matrices) Let $L$ be $a \sigma \times \tau$ (generalized) Löwner matrix with rank $L=q$. Let $\bar{L}$ and $\bar{L}^{*}$ denote the $\sigma \times(\tau+1)$ and $(\sigma+1) \times \tau$ (generalized) Löwner matrices obtained from $L$ by adding one more column or row using some pair $(\bar{x}, \bar{y})$, distinct from the all pairs forming $L$.

(a) If $\sigma=\tau=q=m$ then $(\bar{x}, \bar{y})$ can be chosen so that all (generalized) Löwner submatrices of $\bar{L}$ and $\bar{L}^{*}$ of size $m$ are nonsingular.

(b) If $\sigma=\tau=m>q$ then $(\bar{x}, \bar{y})$ can be chosen so that rank $\bar{L}=q+1$.

(c) If $\sigma=m>q$ and $\tau=m+1$, then $(\bar{x}, \bar{y})$ can be chosen so that rank $\bar{L}^{*}=$ $q+1$.

(d) In (b) and (c), $\bar{L}$ and $\bar{L}^{*}$ contain a singular Löwner submatrix of size $q+1$, for any choice of $(\bar{x}, \bar{y})$. In particular, any Löwner submatrix of size $q+1$ obtained by deleting the $(\tau+1)$ th column of $\bar{L}$, the $(\sigma+1)$ th row of $\bar{L}^{*}$, is singular.

Proof of the extension lemma. Consider the pairs of points

$$
\left(x_{i}, y_{i, j-1}\right) \quad\left(j=1, \ldots, v_{i} ; i=1, \ldots, \theta\right)
$$

$v_{1}+\cdots+v_{\theta}=\tau$. Let

$$
p(x, t):=\frac{y(x)-y(t)}{x-t}, \quad\left[\mathrm{D}_{t}^{j-1} y(t)\right]_{t=x_{i}}:=y_{i, j-1}, \quad\left(j=1, \ldots, v_{i} ; i=1, \ldots, \theta\right),
$$

and

$$
d(x, y):=\left[\sum_{t, j} a_{i, j-1} \mathrm{D}_{t}^{-1} p(x, t)\right]_{t=x_{i}} .
$$

We will show that if the $a_{i j}$ are not all equal to zero, there always exists a pair $(\bar{x}, \bar{y})$ such that

$$
d(\bar{x}, \bar{y}) \neq 0 \text {. }
$$

We can write

where

$$
d(x, y) \prod_{i=1}^{\theta}\left(x-x_{i}\right)^{v_{i}}=y d(x, a)-n(x, a)
$$

$$
a:=\left[\begin{array}{lll}
a_{1}^{\top} & \cdots & a_{\theta}^{\top}
\end{array}\right]^{\top}, \quad a_{i}:=\left[\begin{array}{lll}
a_{i 0} & \cdots & a_{i, v_{t}-1}
\end{array}\right]^{\top}, \quad d(x, a):=p^{\top}(x) \mathbf{a},
$$

and $p(x)$ is defined by $(2.11 \mathrm{c}, \mathrm{d})$. If the polynomial $d(x, a)$ is not identically zero, then (2.23b) can be satisfied. To show this, notice that $p^{\top}(x)=v^{\top} M$, where

$$
v^{\top}=\left[\begin{array}{llll}
x^{m-1} & \cdots & x & 1
\end{array}\right]
$$

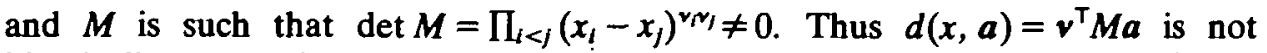
identically zero, unless $a=0$. This implies the existence of $\bar{x}$ such that $d(\bar{x}, a) \neq 0$; (2.23b) is satisfied for any $\bar{y} \neq n(\bar{x}, a) / d(\bar{x}, a)$. 
With the aid of this auxiliary result, we can now prove parts (a)-(d).

(a). $\bar{L}$ and $\bar{L}^{*}$ are obtained by appending to $L$ an additional column or row using $(\bar{x}, \bar{y})$. Let $S_{1}, \ldots, S_{k}$, with $k \leqslant m$, be the $m \times m$ (generalized) Löwner submatrices of $\bar{L}$ which contain the last column (the remaining $m \times m$ Löwner submatrix does not contain the last column and is nonsingular by assumption). The determinants of these submatrices (expanded e.g. with respect to the last column) can be expressed in terms of $d$, defined in (2.23a) for some appropriate set of pairs of points $\left(x_{i}, y_{i, j-1}\right)$; let $d_{i}(\bar{x}, \bar{y}):=\operatorname{det} S_{i}(i=1, \ldots, k)$. Similarly, let $d_{i}^{*}(\bar{x}, \bar{y})(i=1, \ldots, l)$ denote the determinants of the $m \times m$ Lowner submatrices of $\bar{L}^{*}$ which contain the last row. As shown above, the polynomials $d_{i}(i=1, \ldots, k)$ and $d_{i}^{*}(i=1, \ldots, l)$ are not identically zero. Consequently, each one is zero at finitely many points. If we choose $\bar{x}$ different from these finitely many points, then $d_{i}(i=1, \ldots, k)$ and $d_{i}^{*}(i=1, \ldots, l)$, evaluated at $\bar{x}$, will be nonzero. If $\bar{y}$ is chosen different from the finitely many values

$$
\frac{n_{i}(\bar{x}, a)}{d_{l}(\bar{x}, a)} \quad(i=1, \ldots, k), \quad \frac{n_{i}^{*}(\bar{x}, a)}{d_{i}^{*}(\bar{x}, a)} \quad(i=1, \ldots, l),
$$

we obtain the desired result, i.e. $d_{i}(\bar{x}, \bar{y}) \neq 0 \quad(i=1, \ldots, k)$ and $d_{i}^{*}(\bar{x}, \bar{y}) \neq$ $0(j=1, \ldots, l)$.

(b). There exists a $(q+1) \times q$ Löwner submatrix $L^{\prime}$ of $L$, which has full column rank. Using the procedure discussed above, we can append to $L^{\prime}$ an additional column, using an appropriately chosen pair $(\bar{x}, \bar{y})$, such that the augmented matrix, denoted by $L^{\prime}$ has full rank $q+1$. This implies that the rank of $\bar{L}$ is $q+1$.

(c). A $q \times(q+1)$ full row rank Löwner submatrix of $L$ is chosen in this case; the pair $(\bar{x}, \bar{y})$ is such that the augmented $(q+1) \times(q+1)$ matrix is non-singular. Then the rank of $\bar{L}^{*}$ is $q+1$.

(d). This part follows by construction.

Proof of 2.21. From Corollary 2.19 follows that what we are looking for is a Löwner matrix $L_{a}$ that contains $L$ as a submatrix and satisfies $\operatorname{deg} y_{L_{a}}=\operatorname{rank} L_{a}$; equivalently, $L_{a}$ must be such that some full-rank submatrix of $L_{a}$, of size (rank $\left.L_{a}\right) \times\left(\right.$ rank $\left.L_{a}+1\right)$, satisfies the property on the Löwner submatrices given in (2.19b).

By assumption, the rational function attached to $L$ has degree less than rank $L$. Using part (c) of the Extension Lemma, we construct from $L$ the augmented $L^{*}$ by adding one more row, so that rank $\bar{L}^{*}=q+1$. We successively apply parts (b) and (c) of the Extension Lemma $N-2 q-1$ times, i.e. until we obtain an $(N-q) \times(N-q)$ nonsingular (generalized) Löwner matrix. By part (a) of the Extension Lemma we can add one more column such that the resulting $L_{a}$ has the required property, i.e. all $(N-q) \times(N-q)$ Löwner submatrices of $L_{a}$ and $L_{c}^{*}$ are non-singular, which, by Corollary 2.19 , implies that $\operatorname{deg} y_{L_{a}}=\operatorname{rank} L_{a}=N-q$. Hence $y_{L_{\theta}}(x)$ is a rational function of degree $N-q$ interpolating the given $N$ points.

To prove that there exists no function of degree less than $N-q$ interpolating these points, we notice that there are two ways to obtain $L_{a}$ from $L$ : (i) by 
augmenting the rank at each one of the $N-2 q-1$ steps, or (ii) by keeping the rank constant at least during one of these steps. In the first case, by part (d) of the Extension Lemma, at any one of the intermediate steps, no full-rank submatrix can satisfy the required property of Corollary $2.19(\mathrm{~b})$, because each one necessarily contains the new row/column, and the Löwner submatrix obtained by deleting this last row/column at any intermediate step, is singular. In the second case, the required condition of Corollary 2.19(b) cannot be satisfied, a fortiori, not even by $L_{a}$ itself, in contrast to the situation in the first case $y_{L_{a}}$ in the second case does not interpolate all given points).

Thus no interpolating function of degree less than $N-q$ exists.

The following result shows that the rank of $L$ does not depend on the particular partitioning of the $x_{i}$ in the row and column arrays $S$ and $T$ as long as one of them contains a certain number of elements.

2.24 Corollary Suppose that a given (almost) square Löwner matrix $L$ has rank $q$. Then any $k \times(N-k)$ Löwner matrix, with $N-q \geqslant k \geqslant q$ and built from the same data, has the same rank $q$.

Proof. If the given points are interpolated by a function of degree equal to rank $L=q$, then the result follows from Main Lemma 2.5. Thus, we assume that the $N$ points are interpolated by a rational function of least degree $N-q$. Let $B$ be the Bezoutian of such a rational function. By the Main Lemma,

$$
\left[\begin{array}{l}
V_{1} \\
V_{2}
\end{array}\right] B\left[\begin{array}{ll}
W_{1} & W_{2}
\end{array}\right]=\Delta_{V} L_{a} \Delta_{W}
$$

and $\operatorname{rank} B=\operatorname{rank} L_{a}=N-q ; L$ is a submatrix of $L_{a}$; and $V_{1}$ and $W_{1}$ are (possibly generalized) (almost) square Vandermonde matrices built from the $N$ given points in the original order they were chosen to form $L$. Furthermore, $V_{2}$ and $W_{2}$ are also Vandermonde matrices of appropriate size so that the composite matrices pre- and post-multiplying $B$ are square; they contain points interpolated by the rational function in question, distinct from the original $N$ points.

The $N$ original points are re-partitioned in two arbitrary sets of $k$ and $N-k$ elements; the corresponding Vandermonde matrices are denoted by $\bar{V}_{1}$ and $\bar{W}_{1}$; let the remaining $N-2 q$ points form the Vandermonde matrices $\bar{V}_{2}$ and $\bar{W}_{2}$ so that, as before, the composite matrices pre- and post-multiplying $B$ are square. By the Main Lemma

$$
\left[\begin{array}{l}
\bar{V}_{1} \\
\bar{V}_{2}
\end{array}\right] B\left[\begin{array}{ll}
\bar{W}_{1} & \bar{W}_{2}
\end{array}\right]=\Delta_{V} \bar{L}_{a} \Delta_{W},
$$

where $\operatorname{rank} B=\operatorname{rank} \bar{L}_{a}=N-q$.

If the sub-Lowner matrix $\bar{L}$ of $\bar{L}_{a}$ formed from the second partitioning of the original array of points has rank $\bar{q} \leqslant q$ then, by the Extension Lemma 2.22, the

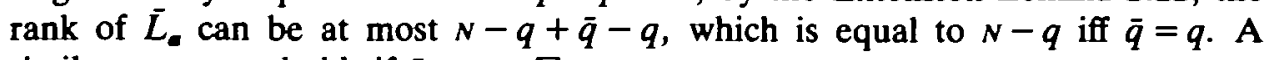
similar argument holds if $\bar{q} \geqslant q$.

Corollary 2.19, Theorem 2.21, and Corollary 2.24 now yield our main result.

2.25 MaIn Theorem Given the array $P$ of $N$ pairs of points, let $L$ be some 
(generalized) Löwner matrix of size $m \times m$ or $m \times(m+1)$, where $m=\frac{1}{2} N$ or $m=\frac{1}{2}(N-1)$. Assume that $\operatorname{rank} L=: q$, and let $y_{L}$ be the (unique) rational function attached to $L$ via (2.11), if it exists.

(a) If $y_{L}$ exists and $\operatorname{deg} y_{L}=q$, then $y_{L}$ is the unique rational function, of least degree $q_{*}=q$, that interpolates all the points in $P$.

(b) Otherwise, there is a family of rational functions of least degree $q_{*}=N-q$, interpolating the given points. This family is parametrized in terms of $2 q_{*}-N+1$ $=N-2 q+1$ parameters.

2.26 THEOREM (the parametrization of all interpolating functions of degree less than $N$ ) If $q_{*}=q$, there exist interpolating functions of degree equal to $q$, and greater than or equal to $N-q$.

If $q_{*}=N-q$, there exist interpolating functions of degrees greater than or equal to $N-q$.

The interpolating function of degree $q$ is unique. There is a family of interpolating functions of degree at most $N-q+\pi-1$, for each $\pi=1, \ldots, q$, parametrized in terms of $N-2 q+2 \pi-1$ parameters, as follows. $L_{\pi}$ denotes some (generalized) Löwner matrix of size $(q-\pi) \times(N-q+\pi)$. Let $\mathscr{C}_{\pi}$ be the set of all column vectors $c_{\pi}$ which satisfy

$$
L_{\pi} c_{\pi}=0, \quad d_{L_{\pi}}\left(x_{i}, c_{\pi}\right) \neq 0 \quad(i=1, \ldots, \theta)
$$

where $d_{L_{x}}$ is defined by $(2.11 b)$, and $x_{i}$, are the distinct points of $P$. The family of all interpolating functions of degree at most $N-q+\pi-1$ is $\left(y_{L_{x}}\left(x, c_{\pi}\right): c_{\pi} \in \mathscr{C}_{\pi}\right)$.

Proof of 2.26. Let $y_{1}(x)$ and $y_{2}(x)$ be two rational functions interpolating the $N$ pairs of points in $P$. It follows that

$$
y_{1}(x)-y_{2}(x)=r(x) \prod_{i=1}^{\theta}\left(x-x_{i}\right)^{v_{i}},
$$

where, as in the proof of Lemma $2.17, r(x)$ is some rational function with poles different from the $x_{i}$. The rational function on the left-hand side of this equation has degree at most $\operatorname{deg} y_{1}+\operatorname{deg} y_{2}$, while the one on the right-hand side has degree at least $N$. If $\operatorname{deg} y_{1}=q$, the inequality $q+\operatorname{deg} y_{2}<N$, implies $r(x)=0$. This shows that $y_{1}$ (if it exists) is the only interpolating function of degree less than $N-q$.

Let $y(x)$ be a rational function interpolating $N$ pairs of points. If $2 \operatorname{deg} y \geqslant N$, a simple count shows that the family of all interpolating functions of degree equal to $\operatorname{deg} y$, has $2 \operatorname{deg} y-N+1$ degrees of freedom. Therefore, the family of all interpolating functions of degree at most $N-q+\pi-1$, is parametrized in terms of $N-2 q+2 \pi-1$ parameters, for $\pi=1, \ldots, q$.

The family of rational functions $y_{L_{z}}$ attached to $L_{\pi}$ via $(2.27 \mathrm{a}, \mathrm{b})$ is a family of interpolating functions of degree at most $N-q+\pi-1$. The Main Theorem guarantees that conditions $(2.27 \mathrm{~b})$ can be satisfied for all $\pi=1, \ldots, q$. The (normalized) $c_{\pi}$ are parametrized in terms of $N-2 q+\pi-1$ parameters. Hence $y_{L_{x}}$ provides a parametrization of all interpolating functions of degree at most $N-q+\pi-1$.

Notice that for $\pi=q$, the matrix $L_{\pi}$ is empty, i.e. condition (2.27a) is empty. 
The only requirement on $c_{\pi}$ in this case is $(2.27 \mathrm{~b})$ which is equivalent to the requirement that each one of its components be nonzero (cf (1.4)).

The next result is concerned with minimal proper rational interpolating functions. Recall (2.12).

2.28 COROLLARY. Under the assumptions of the theorem, the least-degree proper rational function which interpolates the given points is:

(a) If, in addition to the already stated requirement that $\operatorname{deg} y_{L}=q$, the condition $c_{10}+c_{20}+\cdots+c_{Q+1,0} \neq 0$ is satisfied, then $q_{*}=q$.

(b) Otherwise, $q_{*}=N-\dot{q}$. In this case, all such least-degree functions are parametrized as shown in (2.26), where c satisfies the additional constraint

$$
c_{10}+c_{20}+\cdots+c_{\theta 0} \neq 0
$$

$\hat{\theta}$ is the number of distinct points in the column array $\hat{T}$ of $\hat{L}:=L_{\pi}$ for $\pi=1$.

2.29 Discussion (the connection with the realization problem) Let the array $P$ contain a single multiple point of multiplicity $N$, denoted by $\left(x, y_{t-1}\right)(i=$ $1, \ldots, N)$. By $(2.3 b)$, the (almost) square generalized Löwner matrix is given by:

$$
\ell_{i j}=\frac{y_{i+j-1}}{(i+j-1) !}
$$

Clearly, $L$ has Hankel structure.

The corresponding partial realization sequence is $\left(a_{1}, a_{2}, \ldots, a_{N-1}\right)$, where $a_{i}:=y_{i} / i$. The partially defined Hankel matrix defined for the above sequence (see Kalman (1979)) is square of size $N-1$, where:

$$
H_{i j}:=a_{i+j-1} \text { if } i+j<N, \quad H_{i j}:=? \text { otherwise, }
$$

where? stands for undetermined elements conserving the Hankel structure of $H$. We say that $H$ has rank $r$ iff $r$ is the largest positive integer such that the leading $r \times r$ principal submatrix of $H$ is non-singular, independently of the values of the undetermined elements denoted by ? (see Kalman (1979) and Bosgra (1983)).

It follows from the above definitions that $L$ is the principal $m \times m$ or $m \times(m+1)$ submatrix of $H$, according to whether $N$ is even or odd.

If $\operatorname{rank} H=: r \leqslant m$, clearly, $\operatorname{rank} H=\operatorname{rank} L$. If however, $\operatorname{rank} H=r>m$, it can be shown that rank $L=N-r$, and the $m$ th column of $L$ is linearly independent from the previous $m-1$ columns, as predicted by Corollary 2.19(b).

Thus, the problem we have solved reduces to the conventional partial realization problem, if all the $x_{1}$ are the same. This shows that the Lowner matrix is the generalization of the Hankel matrix, when dealing with the general rational interpolation problem.

For a different result on the connection between the realization and the interpolation problems see Audley, Baumgartner, \& Rugh (1975).

2.30 Remarks. (a) Throughout this section we have assumed that both the $x$ and the $y$ values are finite. (a) If at some finite values $x_{i}$, the corresponding $y$ values are infinite, we can write $y(x)=y_{1}(x) y_{2}(x)$, where $y_{1}(x):=\Pi_{i}\left(x-x_{i}\right)^{-1}$ and $y_{2}(x)$ 
is subsequently determined to take care of the remaining (finite) interpolation values. (b) If at infinity, $y=n / d$ is required to be finite, then we must have $\operatorname{deg} n \leqslant \operatorname{deg} d$. (c) If, at infinity, $y$ is infinite, then the interpolation function satisfies $\operatorname{deg} n>\operatorname{deg} d$. Cases (b) and (d) lead to results similar to those of Corollary (2.28). Finally, notice that with values at infinity, some restrictions apply. If $y\left(x_{i}\right)$ is infinite, so are its derivatives at this point, and if $y$ at infinity is finite, so are all its derivatives. Case (b) can also be treated using a bilinear transformation.

(b) From $(2.27 \mathrm{a}, \mathrm{b})$ follows that there are $N-2 q+2 \pi-1$ parameters taking arbitrary values, modulo a set of measure zero consisting of the union of the hyperplanes given by $(2.27 \mathrm{~b})$. The latter are hyperplanes, because by $(2.11 \mathrm{~b})$, $d_{L_{x}}\left(x, c_{x}\right)$ is a linear function of $c_{\pi}$. For $\pi=1$, the linear constraints $(2.27 \mathrm{~b})$ are equivalent to the coprimeness of the numerator and the denominator polynomials of the interpolating function $y_{L}$.

(c) Consider $L$ of size $\sigma \times(N-\sigma)$. Following Lemma 2.17, Remark 2.20b, and Corollary 2.24 , there is a unique rational function attached to any $L$ whenever $q \leqslant \sigma \leqslant m$, where $q$ is the rank of an almost square $L$. The degree of this function is $q$ or less than $q$, according to whether $q_{*}=q$ or $q_{*}=N-q$. In the first case the rational function is also an interpolating function.

If $\sigma<q$, uniqueness is lost, and families of rational functions of degree at most $N-\sigma-1$ are attached to $L$. If these families satisfy $(2.27 \mathrm{~b})$ they become families of interpolating functions; they are parametrized by considering linear combinations of some set of basis vectors for the kernel of the corresponding $L$.

(d) Suppose that $P$ is a symmetric array, i.e. $\left(x_{i}, y_{i}\right)$ is in $P$ implies $\left(x_{i}^{*}, y_{i}^{*}\right)$ is in $P$, where * denotes complex conjugation. Let $n(x, v) / d(x, \delta)$ be some (possibly minimal) interpolating function; $\boldsymbol{v}$ and $\delta$ are the vectors of the numerator and denominator coefficients. It follows that

$$
\frac{n(x, v)+n\left(x, v^{*}\right)}{d(x, \delta)+d\left(x, \delta^{*}\right)}
$$

is a function with real coefficients, interpolating the same array of points $\boldsymbol{P}$.

(e) The classical investigation of the algebraic aspects of the interpolation problem (e.g. the Cauchy interpolation problem, the connection between rational interpolation and continued fractions, etc.) is essentially limited to the generic case, i.e. the case where $2 m+1$ pairs of points are interpolated by a rational function of degree $m$. The investigation of the nongeneric case is concerned with the issue of the so-called inaccessible points. These are the points which are not interpolated by a rational function of degree less than $N-q$, whenever $q_{*}=N-q$. The reader is referred to Belevitch (1970) and Meinguet (1970) for a discussion of these issues.

Some of the results presented in this paper have been discussed in the former reference. In more detail, the Löwner matrix (2.1), Main Lemma 2.5, Corollaries 2.6, 2.19, and 2.24 are developed in Belevitch (1970) in the case of distinct points. The contribution of this section consists mainly of Theorem 2.21, Extension Lemma 2.22. Main Theorem 2.25, and Remark 2.29 on the connection between 
the realization and the interpolation problems. Moreover, all results have been derived in the general case of multiple points.

Various facts concerning square Löwner matrices are given in Fiedler (1984). Further references on the classical aspects of the interpolation problem are Walsh (1935), Shapiro \& Shields (1961). For a recently developed operator-theoretic approach to this area, see Ball (1983).

The main results will now be illustrated in terms of numerical examples.

2.31 Examples (a) Recall example (2.4). Let $x_{1}=0, x_{2}=1, x_{3}=2 ; y_{1}=0$, $y_{20}=0, y_{21}=\frac{1}{2}, y_{22}=0, y_{23}=3, y_{3}=1$. The corresponding generalized Lowner matrix is

$$
L=\left[\begin{array}{ccc}
\frac{1}{2} & 1 & \frac{1}{2} \\
0 & \frac{1}{2} & 0 \\
\frac{1}{2} & 0 & \frac{1}{2}
\end{array}\right]
$$

Clearly, rank $L=2$. Moreover, the condition of Corollary 2.19(b) is satisfied (consider e.g., the $2 \times 3$ submatrix $\bar{L}$ consisting of the first 2 rows). Notice that although the $2 \times 2$ submatrix of $\bar{L}$ consisting of the first and the third columns is singular, Corollary 2.19(b) holds true, because this submatrix is not a Löwner submatrix. Therefore, $y_{L}$ attached to $L$ via $(2.5,6)$ is the desired interpolating function. We have

$$
c=\left[\begin{array}{lll}
c_{10} & c_{20} & c_{21}
\end{array}\right]^{\top}=\left[\begin{array}{lll}
1 & 0 & -1
\end{array}\right]^{\top}
$$

and Example 2.16(a) implies that $y_{L}$ is given by:

$$
1 \frac{y_{L}}{x}-0 \frac{y_{L}}{x}-1\left(\frac{y_{L}}{(x-1)^{2}}-\frac{\frac{1}{2}}{(x-1)}\right)=0
$$

that is,

$$
y_{L}(x)=-\frac{x(x-1)}{2 x^{2}-3 x+1}
$$

Notice that the degree of $y_{L}$ is 2, as predicted by Corollary 2.19 (b).

(b) Let us now take $y_{3}=0$, and the rest as in (a). The corresponding Löwner matrix in this case is:

$$
L=\left[\begin{array}{ccc}
0 & 0 & \frac{1}{2} \\
0 & \frac{1}{2} & 0 \\
\frac{1}{2} & 0 & \frac{1}{2}
\end{array}\right],
$$

which has rank 3. Thus, part (b) of the Main Theorem applies. It predicts that the minimal interpolating function has degree 3. Applying Theorem 2.26 we obtain the parametrization of all interpolating functions of degree 3 , which has one degree of freedom. Let $\mathcal{L}$ have row array $S=\left(x_{2}, x_{2}\right)$, and column array $\hat{T}=\left(x_{1}, x_{2}, x_{2}, x_{3}\right)$. It follows that

$$
L=\left[\begin{array}{cccc}
0 & \frac{1}{2} & 0 & 0 \\
\frac{1}{2} & 0 & \frac{1}{2} & \frac{1}{2}
\end{array}\right]
$$


Let $c:=\left[c_{10}, c_{20}, c_{21}, c_{30}\right]^{\top}$ satisfy $L c=0$; it follows that $c_{20}=0$, and $c_{30}=-c_{10}-$ $c_{21}$; therefore

$$
\begin{aligned}
& c_{10} \frac{y}{x}-0 \frac{y}{x-1}-c_{21}\left[\frac{y}{(x-1)^{2}}-\frac{\frac{1}{2}}{x-1}\right]-\left(c_{10}+c_{21}\right) \frac{y}{x-2}=0 \\
& \text { i.e. } y(x)=\frac{-c_{21} x(x-1)(x-2)}{2\left[c_{21} x^{3}+\left(2 c_{10}-3 c_{21}\right) x^{2}-\left(4 c_{10}-3 c_{21}\right) x-2 c_{10}\right]}
\end{aligned}
$$

The function $y$ interpolates all points, iff conditions (2.27b) are satisfied, i.e.

$$
c_{10} \neq 0, \quad c_{21} \neq 0 \quad c_{10}+c_{21} \neq 0 .
$$

It so happens that the above conditions, in particular $c_{21} \neq 0$, insure the properness of the rational function.

(c) In this case, we let $y_{21}=0, y_{22}=1, y_{23}=1, y_{3}=0$, while the remaining values are as in (a). The corresponding, generalized Löwner matrix is

$$
L=\left[\begin{array}{lll}
0 & 0 & 0 \\
0 & 0 & \frac{1}{2} \\
0 & \frac{1}{2} & \frac{1}{6}
\end{array}\right],
$$

which has rank 2. Unlike case (a) however, the conditions of Corollary 2.19(d) are not satisfied. Hence the second part of the main theorem asserts that the least degree of the interpolating functions is $q_{*}=N-q=6-2=4$. Using Theorem 2.26 we obtain a parametrization, having three degrees of freedom as follows. Let $\hat{L}$ have row array $S=\left(x_{2}\right)$, and column array $\hat{T}=\left(x_{1}, x_{2}, x_{2}, x_{2}, x_{3}\right)$. It follows that

$$
L=\left[\begin{array}{lllll}
0 & 0 & \frac{1}{2} & \frac{1}{6} & 0
\end{array}\right],
$$

which implies that if $c=\left[c_{10}, c_{20}, c_{21}, c_{22}, c_{30}\right]^{\top}$, satisfies $L c=0$, then $c_{22}=-3 c_{21}$. Therefore

that is,

$$
c_{10} \frac{y}{x}+c_{20} \frac{y}{x-1}+c_{21} \frac{y}{(x-1)^{2}}-3 c_{21}\left(\frac{y}{(x-1)^{3}}-\frac{1}{x-1}\right)+c_{30} \frac{y}{x-2}=0,
$$

$y(x)=$

$$
\frac{3 c_{21} x(x-1)^{2}(x-2)}{c_{10}(x-1)^{3}(x-2)+c_{20} x(x-1)^{2}(x-2)}+.
$$

This function interpolates all points, iff conditions $(2.27 \mathrm{~b})$ are satisfied, i.e.

$$
c_{10} \neq 0, \quad c_{21} \neq 0, \quad c_{30} \neq 0 .
$$

For properness, the additional condition

$$
c_{10}+c_{20}+c_{30} \neq 0 \text {. }
$$

must be satisfied. 
(d) Consider the seven pairs of points $\left(0, \frac{1}{2}\right),(1,1),\left(-1,-\frac{1}{2}\right),\left(5, \frac{7}{4}\right),\left(-5, \frac{11}{2}\right)$, $\left(3, \frac{3}{2}\right)$, and $\left(6, \frac{11}{6}\right)$. The corresponding Löwner matrix with row array $T=(0,5,3)$ and column array $S=(1,-1,-5,6)$ is

$$
L_{7}=\left[\begin{array}{cccc}
\frac{1}{2} & 1 & -1 & \frac{2}{9} \\
\frac{3}{16} & \frac{3}{8} & -\frac{3}{8} & \frac{1}{12} \\
\frac{1}{4} & \frac{1}{2} & -\frac{1}{2} & \frac{1}{9}
\end{array}\right]
$$

The rank of $L_{7}$ is one, and conditions of Corollary 2.19(b) are satisfied. Hence $q_{*}=1$. Actually, with

$$
\boldsymbol{c}=\left[\begin{array}{llll}
0 & 0 & 2 & 9
\end{array}\right]^{\top}, \quad L \boldsymbol{c}=0,
$$

we see that (2.16b) implies $y_{L}(x)=(5 x+3) /(2 x+6)$. If the 8th pair $(2,2)$ is added to the set, the Löwner matrix with $S=(0,5,3,2)$ and $T$ as before is

$$
L_{8}=\left[\begin{array}{l}
L_{7} \\
l^{\top}
\end{array}\right], \quad l^{\top}=\left[\begin{array}{llll}
1 & \frac{5}{6} & -\frac{1}{2} & -\frac{1}{24}
\end{array}\right] .
$$

The rank of $L_{8}$ is two, but conditions of Corollary 2.19(b) are not satisfied. Thus with the addition of one more pair, the minimal degree $q_{*}$ jumps from 1 to $8-\operatorname{rank} L_{8}=6$.

\section{Recursiveness of the interpotation problem}

Let $y_{K}(x)$ be a rational function which interpolates the array $P_{K}$ containing $K:=K_{1}+\cdots+K_{\theta}$ (multiple) points $\left(x_{i}, y_{t, j-1}\right)\left(j=1, \ldots, K_{i} ; i=1, \ldots, \theta\right)$. Let $y_{N}(x)=n_{N}(x) / d_{N}(x)$ and $y_{M}(x)=n_{M}(x) / d_{M}(x)$ be rational functions which interpolate the subarrays $P_{N}$ and $P_{M}$ of $P_{K}$, containing $N:=v_{1}+\cdots+v_{\theta}$ and $M:=\mu_{1}+\cdots+\mu_{\theta}$ points, defined by $j=1, \ldots, v_{i}$ and $j=1, \ldots, \mu_{i}$ (both for $i=1, \ldots, \theta)$ respectively; here, $v_{i} \leqslant \kappa_{i}$ and $\mu_{i} \leqslant \kappa_{i}(i=1, \ldots, \theta)$. Notice that if $v_{i}$ or $\mu_{i}$ is zero, then the simple point $\left(x_{i}, y_{i 0}\right)$ is not interpolated by $y_{N}(x)$ or $y_{M}(x)$, as the case may be.

The first step towards a theory of recursive minimal interpolation is to express $y_{K}$ as a function of $y_{N}$ and $y_{M}$. To that end we define the rational functions

$$
p(x):=\prod_{i=1}^{\theta}\left(x-x_{i}\right)^{v_{i}-\mu_{4}}, \quad p_{j}(x):=\prod_{i \neq j}\left(x-x_{i}\right)^{v_{i}-\mu_{l}},
$$

and the rational function $s(x)$ which satisfies the following interpolation conditions at each $x_{i}(i=1, \ldots, \theta)$. If

$$
0 \leqslant \mu_{i} \leqslant v_{i} \leqslant \kappa_{i},
$$

then

$$
\begin{array}{r}
D^{\prime} s\left(x_{i}\right)=\frac{j !}{\left(j+v_{i}-\mu_{i}\right) !}\left[D^{j+v_{i}-\mu_{i}}\left(-\frac{1}{p_{i}(x)} \frac{d_{N}(x)}{d_{M}(x)} \frac{y_{K}(x)-y_{N}(x)}{y_{K}(x)-y_{M}(x)}\right)\right]_{x=x_{i}} \\
\left(j=0,1, \ldots, \kappa_{i}-v_{i}-1\right),
\end{array}
$$


where $D^{\prime} f\left(x_{i}\right)$ denotes the $j$ th derivative of the function $f(x)$ with respect to $x$, evaluated at $x=x_{i}$; further,

$$
s\left(x_{i}\right) \neq-\frac{1}{p\left(x_{i}\right)} \frac{d_{N}\left(x_{i}\right)}{d_{M}\left(x_{i}\right)} \text { if } v_{i}=\mu_{i} .
$$

The interpolation conditions at those points $x_{i}$ satisfying $0 \leqslant v_{i} \leqslant \mu_{i} \leqslant \kappa_{i}$ are defined similarly.

3.2 LEMMA With $p(x)$ and $s(x)$ defined as above, the following holds true:

$$
y_{K}(x)=\frac{n_{M}(x)+n_{M}(x) p(x) s(x)}{d_{N}(x)+d_{M}(x) p(x) s(x)} .
$$

Computations similar to those involved in the proof of the above lemma are carried out in the proof of Theorem 3.9; the proof of Lemma 3.2 is thus omitted. We just note that condition (3.1d) guarantees that no one of the points interpolated by both $y_{N}$ and $y_{M}$ is a common root of the numerator and the denominator of (3.3).

The above considerations show that the problem of determining a function interpolating the points in $P_{K}$, given two nonidentical functions interpolating subarrays $P_{N}$ and $P_{M}$ thereof, can be reduced to an interpolation problem which involves the additional points only (cf. (3.1c)). This is achieved with the aid of the linear fractional representation formula (3.3).

Our next goal is to introduce minimality in the above considerations. For the remainder of this section we will assume that $v_{i} \geqslant \mu_{i}(i=1, \ldots, \theta)$ and $K=N+1$; this means that $P_{M}$ is a subarray of $P_{N}$ and $P_{K}$ contains one pair of points more than $P_{N}$. Theorem (3.9) shows how the linear fractional representation formula (3.3) yields a minimal updating (when the additional point is simple or multiple) provided $y_{M}$ is chosen appropriately. The first step towards this goal is

3.4 THEOREM Let $y_{N-1}(\pi ; x)$ be a parametrization of all minimal-degree interpolating functions of the $N-1$ points $P_{N-1}$, in terms of some vector parameter $\pi$, of appropriate dimension. Let $y_{N}\left(\omega_{0} ; x\right)$ denote some minimal-degree interpolating function of the $N$ points $p_{N}$. A parametrization $y_{N}(\sigma ; x)$ of all minimal-degree interpolating functions of the $N$ points, in terms of the vector parameter $\sigma$, is obtained as follows.

(a) If $\operatorname{deg} y_{N}=\operatorname{deg} y_{N-1}$, then $y_{N}(\sigma ; x)=y_{N-1}(\overline{\boldsymbol{\pi}} ; x)$, where $\sigma:=\overline{\boldsymbol{\pi}}$ is obtained by appropriately restricting $\pi$; the vector $\pi$ has either one degree of freedom more than $\bar{\pi}$ or is empty.

(b) If $\operatorname{deg} y_{N}>\operatorname{deg} y_{N-1}$, we have

$$
y_{N}(\sigma ; x)=\frac{n_{N}\left(\sigma_{0}, x\right)+n_{N-1}(\pi ; x) p(\tau ; x)}{d_{N}\left(\sigma_{0} ; x\right)+d_{N-1}(\pi ; x) p(\tau, x)}, \quad \sigma:=(\pi, \tau),
$$

where $p(\tau ; x)=\left(x-x_{j}\right) \hat{p}(\tau ; x)$; also $x_{j}$ is the additional point contained in $P_{N}$, while $\hat{p}(\tau ; x)$ is an arbitrary polynomial of degree $\operatorname{deg} \hat{p}=\operatorname{deg} y_{N}-\operatorname{deg} y_{N-1}-1$, 
and the parameters $\pi$ and $\boldsymbol{\tau}$ satisfy

$$
d_{N}\left(\omega_{0} ; x_{i}\right)+d_{N-1}\left(\pi ; x_{l}\right) p\left(\tau ; x_{i}\right) \neq 0,
$$

with $i$ ranging over the distinct points in $P_{N-1}$. Moreover $\tau$ can be chosen so that the numerator and the denominator of $y_{N}(\sigma ; x)$ depend affinely on $\sigma$, provided that numerator and denominator of $y_{N-1}(\pi ; x)$ depend affinely on $\pi$.

For the proof of the Theorem we will use

3.6 Proposition Let $y_{N}$ be a minimal-degree rational function interpolating $N$ pairs of points; a corresponding (almost) square Löwner matrix will be denoted by $L_{N}$. Given one additional pair to be interpolated, let $L_{N+1}$ denote an (almost) square Löwner matrix constructed from all $N+1$ pairs of points.

(a) If $y_{N}$ is unique, i.e. $2 \mathrm{deg} y_{N}<N$, it interpolates the additional point if and only if rank $L_{N+1}=\operatorname{rank} L_{N}$.

(b) Suppose that $y_{N}$ is nonunique, i.e. $2 \operatorname{deg} y_{N} \geqslant N$; let $y_{N}(\sigma ; x)$ be a parametrization of all minimal-degree rational functions interpolating the given $N$ points. The additional point is interpolated by $y_{N}\left(\omega_{0} ; x\right)$, for some value of the vector parameter $\sigma=\sigma_{0}$, if and only if rank $L_{N+1}=\operatorname{rank} L_{N}+1$. Otherwise, the minimal degree of a rational function interpolating all $N+1$ points is $\operatorname{deg} y_{N}+1$.

Proof of 3.6. (a). This is a consequence of Main Lemma 2.5. (b) From the proof of Theorem 2.21 as well as Main Theorem 2.25(b), it follows that if $y_{N}$ is nonunique and rank $L_{N+1}=\operatorname{rank} L_{N}$, then the minimal degree of a rational function interpolating all $N+1$ points is $\operatorname{deg} y_{N}+1$.

Proof of 3.4. (a) In the case of degree equality, if $y_{N-1}(x)$ is unique, we have $y_{N}(\sigma ; x)=y_{N}(x)=y_{N-1}(x)$, i.e. $\sigma$ is empty. If $y_{N-1}(\pi ; x)$ is nonunique, by Proposition 3.6(b), the $N$ th point is interpolated by $y_{N}(\sigma ; x):=y_{N-1}(\bar{\pi} ; x)$, by appropriately restricting $\pi$ to $\overline{\boldsymbol{\pi}}$.

(b1) Let $y_{N-1}$ be unique. By Main Theorem 2.25, the minimal degree of the rational functions interpolating all $N$ points is $N$ minus the rank of the corresponding (almost) square Löwner matrix, i.e. $\operatorname{deg} y_{N}=N-\left(\operatorname{deg} y_{N-1}+1\right)$, which can be rewritten as

$$
\operatorname{deg} y_{N}+\operatorname{deg} y_{N-1}=N-1 \text {. }
$$

This implies that $\operatorname{deg} \hat{p}=2 \operatorname{deg} y_{N}-N$; since $\hat{p}$ is completely arbitrary, it has $2 \operatorname{deg} y_{N}-N+1$ degrees of freedom, subject to restrictions (3.5b). Therefore $\boldsymbol{\sigma}=\boldsymbol{\tau}$, and

$$
y_{N}(\sigma ; x)=\frac{n_{N}\left(\sigma_{0} ; x\right)+n_{N-1}(x)\left(x-x_{j}\right) \hat{p}(\sigma ; x)}{d_{N}\left(\sigma_{0} ; x\right)+d_{N-1}(x)\left(x-x_{j}\right) \hat{p}(\sigma ; x)}
$$

interpolates all $N$ points. Furthermore, it has $\operatorname{deg} \hat{p}+1=2 \operatorname{deg} y_{N}-N+1$, parameters, which by Main Theorem 2.25(b), is the correct number of parameters in this case. Hence $y_{N}(\sigma ; x)$ provides the desired parametrization, while numerator and denominator depend affinely on $\sigma$.

(b2) Let $y_{N-1}(\pi ; x)$ be nonunique. By Proposition 3.6(b), $\operatorname{deg} y_{N}=\operatorname{deg} y_{N-1}+$ 1 , and $y_{N}$ contains one more degree of freedom than $y_{N-1}$. If we let 
$f(\tau ; x)=1 / \tau$, where $\tau$ is a nonzero parameter:

$$
y_{N}(\sigma ; x)=\frac{\tau n_{N}\left(\sigma_{0} ; x\right)+n_{N-1}(\pi, x)\left(x-x_{j}\right)}{\tau d_{N}\left(\omega_{0} ; x\right)+d_{N-1}(\pi, x)\left(x-x_{j}\right)},
$$

provides the desired parametrization, with (3.5b) holding true. Notice again that numerator and denominator depend affinely on $\sigma=(\pi, \tau)$, provided that numerator and denominator of $y_{N-1}(\pi ; x)$ depend affinely on $\pi$.

We now turn our attention to the problem of minimally updating a minimaldegree interpolating function, in order to take care of the additional point $\left(x_{j}, y_{j v}\right)$, which is simple or multiple according to whether $v_{j}=0$ or $v_{j}>0$. To obtain a minimal-degree interpolating function $y_{N+1}$, using the linear fractional representation (3.3), we choose $M, y_{M}, y_{N}$ as follows:

(3.8) $M$ is the largest positive integer less than $N$, for which there exist minimal-degree interpolating functions $y_{N}, y_{M}$ satisfying $y_{N}(x) \neq y_{M}(x)$.

The main result of this section is the following.

3.9 THEOREM Let $y_{N}(x)$ and $y_{M}(x)$ satisfy (3.8). Let also $y_{N}(\sigma ; x)$ be $a$ parametrization of all minimal-degree interpolating functions of the $N$ points in $P_{N}$. Consider the $(N+1)$ th interpolation point $\left(x_{j}, y_{j v_{i}}\right)$.

(a) If $\operatorname{deg} y_{N+1}=\operatorname{deg} y_{N}$, then $y_{N+1}(x)=y_{N}\left(\omega_{0} ; x\right)$ provides the desired minimal updating.

(b) If $\operatorname{deg} y_{N+1}>\operatorname{deg} y_{N}$, the linear fractional representation (3.3) provides a rational function of minimal degree interpolating all the $N+1$ points, provided that (i) $s(x)$ is constant, if $s\left(x_{j}\right) \neq s\left(x_{i}\right)$, for those $x_{i}$ satisfying $v_{i}=\mu_{i}$, and (ii) $s(x)=\alpha /(x+\beta)$, if $s\left(x_{j}\right)=s\left(x_{i}\right)$ for some $x_{i}$ with $v_{i}=\mu_{i}$, where

$$
\begin{aligned}
& s\left(x_{i}\right) \neq-\frac{1}{p\left(x_{i}\right)} \frac{d_{N}\left(x_{i}\right)}{d_{M}\left(x_{i}\right)}, \text { for all } x_{i} \text { satisfying: } v_{i}=\mu_{i} \text { for } i=1, \ldots, \theta, \quad \text { (3.10a) } \\
& s\left(x_{j}\right)=-\frac{1}{p\left(x_{j}\right)} \frac{d_{N}\left(x_{j}\right)}{d_{M}\left(x_{j}\right)} \frac{y_{j 0}-y_{N}\left(x_{j}\right)}{y_{j 0}-y_{M}\left(x_{j}\right)}, \quad \text { if } x_{j} \text { is simple, i.e. } v_{j}=\mu_{j}=0 . \\
& s\left(x_{j}\right)=-\frac{\mu_{j} !}{v_{j} ! p_{j}\left(x_{j}\right)} \frac{d_{M}\left(x_{j}\right)}{d_{M}\left(x_{j}\right)} \frac{y_{j v_{j}}-\mathrm{D}^{\left(v_{j}\right)} y_{N}\left(x_{j}\right)}{y_{j, j}-\mathrm{D}^{\left(\mu_{j}\right)} y_{M}\left(x_{j}\right)}, \quad \text { if } x_{j} \text { is multiple, i.e. } v_{j} \geqslant \mu_{j} \geqslant 0 .
\end{aligned}
$$

The function $s(x)$ is also of minimal degree.

The theorem shows that the determination of $y_{N+1}$ is reduced to the determination of a rational function $s(x)$ of degree at most one; the value of $s(x)$ at $x=x_{j}$ is specified, while its value at points $x=x_{i}$ such that $v_{i}=\mu_{i}$, has to be different from further specified values.

Proof. (a) The procedure given in Theorem 3.4 is followed. In order to check whether the degree of $y_{N}$ is equal to the degree of $y_{N+1}$, we consider

$$
\left[D^{v_{j}+1}\left[y_{N}(x) d_{N}(\sigma ; x)-n_{N}(\sigma ; x)\right]\right]_{x=x_{j}}=0 \text {, where }\left[D^{i} y(x)\right]_{x=x_{j}}=y_{j i} \text {. }
$$


(It should be remarked that this condition is easy to check only if the parametrization is affine.) If this equation is not in conflict with one of the relationships in (3.5b), we have equality of the two degrees; otherwise the degree of $y_{N+1}$ increases.

(b) The rational function $s(x)$ satisfying $(3.10 \mathrm{a}, \mathrm{b}, \mathrm{c})$ can have minimal degree one or zero, according to whether $s\left(x_{j}\right)$ is equal to some $s\left(x_{l}\right)$ in (3.10a), or not. This proves the minimality of $s$. Assuming that $(3.10 \mathrm{~b}, \mathrm{c})$ hold true, we will next prove the minimality of $\operatorname{deg} y_{N+1}$.

(b1) Let $y_{N}$ be non-unique, i.e. $2 \operatorname{deg} y_{N} \geqslant N$. In this case $M=N-1$. By Proposition 3.6b deg $y_{N}=\operatorname{deg} y_{N-1}+1$, and $y_{N}(x)$ interpolates one more point than $y_{N-1}(x)$. Hence $p(x)=x-x_{j}$, which implies $\operatorname{deg} y_{N}=\operatorname{deg}\left(y_{N-1} p\right)$. Again by Proposition $3.6 \mathrm{~b}, \operatorname{deg} y_{N+1}=\operatorname{deg} y_{N}+1$. Therefore $s(x)$ has to be of degree one (otherwise $\operatorname{deg} y_{N+1}=\operatorname{deg} y_{N}$, which is a contradiction). Moreover, any $s(x)$ of degree one will result in a minimal-degree $y_{N+1}$.

(b2) Let $y_{N}$ be unique, i.e. $2 \mathrm{deg} y_{N}<N$. By assumption (3.8), $y_{N}=y_{i}$ for $i=M+1, \ldots, N-1$ (which implies that the latter interpolating functions are also unique), while $y_{M}$ is no longer unique; however, from (3.8) and Main Theorem 2.25 it follows that $\operatorname{deg} y_{M}=\operatorname{deg} y_{N}$. By Main Theorem 2.25(b):

$$
2 \operatorname{deg} y_{N}=2 \operatorname{deg} y_{M}=M \text {. }
$$

Clearly, $y_{N}$ interpolates $N-M$ points more than $y_{M}$. This implies that $\operatorname{deg} p=$ $N-M$. Finally by $(2.25 \mathrm{~b})$, the degree of $y_{N+1}$ is

$$
N+1-\left(\operatorname{deg} y_{N}+1\right)=N-\operatorname{deg} y_{N} \Rightarrow \operatorname{deg} y_{N+1}=\operatorname{deg}\left(p y_{M}\right) \text {. }
$$

In this case, if the minimal degree of $s(x)$ turns out to be one, we have to choose $s(x)=\alpha /(x+\beta)$, and not $s(x)=\alpha x+\beta$, since the latter would cause the degree of $y_{N+1}$ to be non-minimal (see Remark 3.13f). Of course, $s(x)$, contrary to the case (b1), might turn out to be a constant.

Minimality having been settled, there remains to show that expressions (3.10b) and $(3.10 \mathrm{c})$ hold true. The first one follows readily from (3.3). In order to prove (3.10c) we proceed as follows.

Solving (3.3) with respect to $r:=p s$, we obtain $q r=r$, where

$$
q(x):=d_{M}(x)\left(y_{N+1}(x)-y_{M}(x)\right), r(x):=d_{N}(x)\left(y_{N+1}(x)-y_{N}(x)\right) \text {. }
$$

With $\mathrm{D}$ denoting derivation with respect to $x$, we have

$$
\mathrm{D}^{\boldsymbol{x}} q=\sum_{i=0}^{\kappa} \gamma_{\alpha d} \mathrm{D}^{\kappa-i} d_{M}\left(\mathrm{D}^{\prime} y_{N+1}-\mathrm{D}^{\prime} y_{M}\right) \text {, where } \gamma_{\boldsymbol{\alpha} i}:=\frac{\boldsymbol{\kappa} !}{(\kappa-i) ! i !} .
$$

If in the above expression the subscript $M$ is replaced by $N$, we obtain the $K$ th derivative of $r$. Taking $m-1:=v_{j}$ successive derivatives of the equation $q r=r$ we obtain the system of equations

$$
Q \boldsymbol{r}=\boldsymbol{\rho}
$$

where $Q$ is an $m \times m$ lower triangular matrix with $Q_{j}=\gamma_{i-1, j-1} D^{\prime-j} Q$ for $j \leqslant i$, and $r$ and $\rho$ are $m \times 1$ column vectors with $D^{i-1} r$ and $D^{-1} r$ as $i$ th entry, respectively. 
Let us consider point $x_{j}$ and the resulting restrictions for $r$ and its derivatives at $\boldsymbol{x}=\boldsymbol{x}_{\boldsymbol{j}}$. By assumption:

$$
\begin{aligned}
& \mathrm{D}^{\lambda-1} y_{M}\left(x_{j}\right)=\mathrm{D}^{i-1} y_{N}\left(x_{j}\right)=y_{t, j-1} \quad\left(i=1, \ldots, \mu_{j}\right), \quad \mathrm{D}^{\mu / y_{M}} \neq y_{j \mu^{\prime}}, \\
& \mathrm{D}^{i-1} y_{N}\left(x_{j}\right)=y_{j, i-1} \quad\left(i=\mu_{j}+2, \cdots, v_{j}\right) \text {. }
\end{aligned}
$$

These relationships imply $D^{\prime-1} q\left(x_{j}\right)=0\left(i=1, \ldots, \mu_{j}\right), \quad D^{\mu /} q\left(x_{j}\right) \neq 0$, and $\mathrm{D}^{i-1} r\left(x_{j}\right)=0\left(i=1, \ldots, v_{j}\right)$. The system (3.11) contains $v_{j}+1$ equations. The first $\mu_{j}$ ones are of the form zero equals zero. The next $v_{j}-\mu_{j}$ are of the form: $\mathrm{D}^{\mu_{i}} q\left(x_{j}\right) \mathrm{D}^{i-1} r\left(x_{j}\right)=0$ for $i=1, \ldots, v_{j}-\mu_{j}$; they imply $\mathrm{D}^{\prime} r\left(x_{j}\right)=0$, for the same indices $i$. These considerations prove that $p$ is a polynomial having the form given in (3.1a). If $v_{i}>\mu_{i}$, then $r\left(x_{i}\right)=0$. Thus the numerator and denominator of $y_{N+1}$ in (3.3) cannot have $x-x_{i}$ as a common factor, and therefore, $y_{N+1}$ interpolates $\left(x_{i}, y_{i, m-1}\right)$ for $m=1, \ldots, v_{i}$. If $v_{i}=\mu_{i}$, to prevent $x-x_{i}$ from being a common factor, we require (3.10a) to hold.

The last equation in (3.11) turns out to be

$$
\gamma_{v_{j, \mu}, D^{\mu}} \mathrm{D}^{\mu} q\left(x_{j}\right) \mathrm{D}^{v_{j}-\mu_{j}} r\left(x_{j}\right)=\mathrm{D}^{\nu_{j}} r\left(x_{j}\right) .
$$

Since, by (3.1a), we have $r(x)=\left(x-x_{j}\right)^{v_{j}-\mu_{j}} p_{j}(x) s(x)$, we obtain

$$
\mathrm{D}^{v_{j}-\mu_{i}} r\left(x_{j}\right)=\left(v_{j}-\mu_{j}\right) ! p_{j}\left(x_{j}\right) s\left(x_{j}\right)
$$

which, together with (3.12), implies (3.10c).

This completes the proof of the theorem.

3.13 Remarks. (a) If $N+t$ new points are provided, with $t>1$, a $t$-step updating of the interpolation function $y_{N}$ is obtained by performing $t$ successive one-step updatings as shown in Theorem 3.9.

(b) If in Lemma (3.2), for some of the new points to be interpolated, we have

$$
y_{N}\left(x_{i}\right)=y_{M}\left(x_{i}\right) \neq y_{i 0} \text {, }
$$

then the function $y_{K}$ cannot interpolate at that point. This can be avoided by appropriate choice of the various functions involved; compare e.g. Theorem 3.9, where this situation cannot occur.

(c) From $(3.10 \mathrm{a}-\mathrm{c})$ we conclude that the rational function $s(x)$ does not depend on $y_{j i}$, except for $i=\mu_{j}$ and $i=v_{j}$. The updating, therefore, depends on the new values at the $(H+1)$ th and $(N+1)$ th steps.

(d) If, in formula (3.3), $s(x)$ is allowed to be an arbitrary rational function satisfying $(3.10 \mathrm{a}-\mathrm{c})$ (i.e. not necessarily of minimal degree as in Theorem 3.9), then we obtain a parametrization of all rational functions interpolating the given $N+1$ points.

(e) The treatment in this section was inspired by the recursiveness approach as applied to the problem of partial realizations. For details, see Antoulas (1985).

(f) In the proof of Theorem (3.9), it should be noticed that in case (b2) not all $s(x)$ 's of minimal degree satisfying (3.10b) give rise to $y_{N+1}$ 's of minimal degree. Only the indicated choice has that property. Thus to every minimal $y_{N+1}$ there corresponds a minimal $s$; the converse is not true. 
(g) One of the main advantages of the recursiveness considerations is that the use of the Löwner matrix is circumvented. Consequently, one does not have to compute the rank of matrices, whose size increases with the data.

(h) Recall (3.8). Clearly, $y_{N}$ interpolates any subset of the given $N$ points. For minimality of $y_{N+1}$ however, we have to choose $y_{M}$ different from $y_{N}$, but of degree as close to the degree of $y_{N}$ as possible.

(i) A special case of the linear fractional representation formula (3.3) used for recursive interpolation of distinct points can be found in Walsh (1935, Chapter $\mathrm{X})$. Minimality however is not obtained.

An example will now illustrate the recursiveness aspects of the interpolation problem.

3.14 EXAMPLE The procedure to follow will first be summarized. At the $N$ th step we compute $y_{N}(\sigma ; x)$ and $y_{M}$ so as to satisfy (3.8). If $\operatorname{deg} y_{N+1}=\operatorname{deg} y_{N}$, we restrict $\sigma$ appropriately. If the degree increases however, we first use Theorem 3.9(b) to compute one $(N+1)$ th minimal updating, and then Theorem 3.4 to obtain a parametrization of all $(N+1)$ th minimal updatings. The last two steps can be combined in one. We prefer not to do so however, because in this case the numerator and the denominator will not depend affinely on the parameters; additional work will be needed to achieve this.

The points $\left(x_{i}, y_{i j}\right)$ to be recursively interpolated are: $(0,0) ;(1,0) ;(2,1) ;(4,2)$; $(3,0) ;(6,3) ;(-1,-8) ;(0,0) ;(0,0)$; the second and the third $(0,0)$ pairs are to be interpreted as specifying the values of the first and of the second derivatives at zero. At the first and the second steps: $y_{1}=y_{2}=0$. At the third step, a parametrization of all minimal interpolating functions is obtained using Main Theorem 2.25 and Theorem 2.26:

$$
y_{3}(\alpha, \beta ; x)=\frac{x(x-1)}{(\alpha+\beta+1) x^{2}-(2 \alpha+3 \beta+1) x+2 \beta},
$$

where

$$
\alpha \neq 0, \quad \beta \neq 0 .
$$

For the fourth step, we notice that $(4,2)$ is interpolated by the above expression iff

$$
4 \alpha+3 \beta+3=0 \text {. }
$$

Since (3.16) is not in contradiction with (3.15), we are in case (a) of Theorem 3.9. Thus

$$
\begin{gathered}
y_{4}(\alpha ; x)=\frac{3 x(x-1)}{-\alpha x^{2}+6(\alpha+1) x-2(4 \alpha+3)}, \\
\alpha \neq 0, \quad 4 \alpha+3 \neq 0 .
\end{gathered}
$$

For the fifth step we notice that $y_{4}(\alpha ; x)$ cannot interpolate $(3,0)$, for any finite value of $\alpha$. Thus, the degree increases. We apply Theorem 3.9(b) to obtain

$$
y_{5}(x)=\frac{n_{4}(-1 ; x)+n_{3}(-2,1 ; x)(x-4) s(x)}{d_{4}(-1 ; x)+d_{3}(-2,1 ; x)(x-4) s(x)} .
$$


Conditions $(3.10 \mathrm{a}, \mathrm{b})$ are as follows:

$$
s(0) \neq \frac{1}{4}, s(1) \neq \frac{1}{2}, s(2) \neq \frac{3}{2}, s(3)=3 .
$$

The minimal-degree rational function satisfying $(3.17 \mathrm{~b})$ is

$$
s(x)=3 \text {. }
$$

This implies that

$$
y_{5}(x)=\frac{3 x(x-1)(x-3)}{x^{2}+6 x-22} .
$$

Applying Theorem 3.4 we obtain a parametrization of all $y_{5}$ 's.

$$
\begin{aligned}
y_{5}(\alpha, \beta ; x) & =\frac{\beta n_{5}(x)+n_{4}(\alpha ; x)(x-3)}{\beta d_{5}(x)+d_{4}(\alpha ; x)(x-3)} \\
= & \frac{3(\beta+1) x(x-1)(x-3)}{-\alpha x^{3}+(9 \alpha+\beta+6) x^{2}+(-26 \alpha+6 \beta-24) x+6(4 \alpha+3)-22 \beta} .
\end{aligned}
$$

Restrictions (3.5b) for the points $0,1,2,4,3$, respectively, turn out to be

$$
12 \alpha-11 \beta+9 \neq 0, \quad 2 \alpha-5 \beta \neq 0, \quad \beta+1 \neq 0, \quad \beta+1 \neq 0, \quad 5 \beta-36 \neq 0 .
$$

For the interpolation of $(-1,-8)$ at the sixth we obtain from (3.17e) the relationship

$$
4 \alpha-2 \beta+3=0
$$

Since (3.18a) is not in contradiction with any of the relationships in (3.17f), we conclude that the minimal degree does not increase. We apply Theorem 3.9 a to obtain

$$
y_{6}(\alpha ; x)=\frac{3\left(2 \alpha+\frac{5}{2}\right) x(x-1)(x-3)}{-\alpha x^{3}+\left(11 \alpha+\frac{15}{2}\right) x^{2}-\left(14 \alpha+\frac{45}{2}\right) x-(20 \alpha+15)} .
$$

Combining (3.18a) and (3.17f) the following restrictions are obtained

$$
\alpha \neq-\frac{3}{4}, \alpha \neq-\frac{15}{16}, \alpha \neq-\frac{5}{4}, \alpha \neq \frac{57}{20} \text {. }
$$

Formula (3.18b) interpolates $(6,3)$ iff the parameter $\alpha$ has the value

$$
\alpha=-\frac{15}{20} \text {. }
$$

Since (3.19) is not in contradiction with (3.18c), for the second consecutive step, the minimal degree remains. We have

$$
y_{7}(x)=\frac{7 x(x-1)(x-3)}{x^{3}+2 x^{2}-12 x-6}
$$

this is the unique rational function of minimal degree interpolating the first seven points of our list. 
Since the derivative of $y_{7}$ at zero is not zero, in order to interpolate the eighth point, the degree of the interpolating function will have to increase. We apply Theorem 3.9(b) to obtain

$$
y_{8}(x)=\frac{n_{7}(x)+n_{6}(0 ; x)(x-6) s(x)}{d_{7}(x)+d_{6}(0 ; x)(x-6) s(x)}
$$

Conditions $(3.10 \mathrm{a}, \mathrm{c})$ yield the following conditions on $s(x)$.

$$
s(0) \neq \frac{1}{2}, s(1) \neq-1, s(3) \neq 1, s(2) \neq \frac{7}{4}, s(4) \neq \frac{7}{2}, s(-1) \neq 1 \text {, and } s(0)=\frac{7}{6} .
$$

This implies that the minimal degree of $s(x)$ is zero: $s(x)=?$.

One minimal-degree interpolating function for all eight points is therefore

$$
y_{8}(x)=\frac{7 x^{2}(x-1)(x-3)}{13 x^{3}-44 x^{2}-2 x+48}
$$

Using Theorem 3.4 we also obtain a parametrization of all $y_{8}$ 's:

$$
y_{8}(\alpha ; x)=\frac{n_{8}(x)+\alpha x n_{7}(x)}{d_{8}(x)+\alpha x d_{7}(x)} .
$$

Conditions (3.5b) yield the following restrictions for $\alpha$ :

$$
\alpha \neq 1, \alpha \neq \frac{1}{3}, \alpha \neq-1 .
$$

In order for the second derivative of the function to vanish at zero we must have in (3.20a) that $\alpha=-1$. This is in contradiction with (3.20b). Hence the degree increases again. $y_{9}$ can be expressed as follows:

$$
y_{9}(x)=\frac{n_{8}(0 ; x)+n_{7}(x) x s(x)}{d_{8}(0 ; x)+d_{7}(x) x s(x)} .
$$

Conditions $(3.10 \mathrm{a}, \mathrm{c})$ yield:

$s(1) \neq 1, \quad s(3) \neq \frac{1}{3}, \quad s(2) \neq-1, \quad s(4) \neq-1, \quad s(-1) \neq-1, \quad s(6) \neq-1, \quad s(0)=-1$.

It follows that $s(x)$ has minimal degree one. In this case, like in (b1) of the proof of Theorem 3.9, any minimal $s(x)$ will do. We choose $s(x)=x-1$. The resulting minimal degree interpolating function is

$$
y_{9}(x)=\frac{7 x^{3}(x-1)(x-3)}{x^{5}+x^{4}-x^{3}-38 x^{2}+4 x+48} .
$$

It is interesting to notice that in (3.21a) if we choose $s(x)=-1$, four of the conditions (3.21b) are violated. This means that four pole-zero cancellations occur in (3.21a). Since the function has degree four, no more pole-zero cancellations can take place. The resulting function should be a constant and it should interpolate all remaining five points. Actually, $s(x)=0$, which interpolates all but the four points at which the pole-zero cancellation occurred, namely $x=2,4,-1,6$. This situation is as predicted by Corollary 2.19 . Finally, by (2.1) and $(2.3 \mathrm{a}, \mathrm{b})$ the generalized Löwner matrix of the nine points, with row array 
$S=(0,3,6,-1)$, and column array $T=(0,0,1,2,4)$, is

$$
L=\left[\begin{array}{ccccc}
0 & 0 & 0 & \frac{1}{2} & \frac{1}{2} \\
0 & 0 & 0 & -1 & 2 \\
\frac{1}{2} & \frac{1}{12} & \frac{3}{3} & \frac{1}{2} & \frac{1}{2} \\
-8 & -8 & 4 & 3 & 2
\end{array}\right]
$$

The rank of this matrix is four, but the conditions of Corollary (2.19b) are not satisfied. Thus by Main Theorem 2.25(b), the degree of the resulting minimal interpolating functions is $9-4=5$, which is the same as the degree of $y_{9}(x)$ in (3.21c).

\section{REFERENCES}

Artken, A. C. 1964 Determinants and Matrices. Interscience, New York.

Anderson, B. D. O., \& JuRy, E. I. 1976 Generalized Bezoutian and Sylvester matrices in multivariable linear control. IEEE Trans. autom. Control, AC-21, 551-556.

ANDerson, B. D. O., \& LinNemanN, A. 1985 Control of decentralized systems with distributed complexity. Proc. 24th, IEEE CDC, Ft. Lauderdale, 24, pp. 1468-1472.

Antoulas, A. C. 1985 On recursiveness and related topics in linear systems. Technical Report TR 8507, Department of Electrical and Computer Engineering, Rice University; IEEE Trans. autom. Control, 31, Dec. 1986.

Audley, D. R., Baumaartiner, S. L., \& RuGH, W. J. 1975 Linear system realization based on data set representations. IEEE Trans. autom. Control; AC-20, 432-433.

BALL, J. A. 1983 Interpolation problems of Pick-Nevanlinna and Loewner types for meromorphic matrix functions, Integral $E q$. Oper. Theor, 6, 804-840.

Belevitch, V. 1970 Interpolation Matrices. Philips Res. Reports 25, pp. 337-369.

BosGRA, O. H. 1983 On parametrizations for the minimal partial realization problem. Syst. and Control Lett., 3, 181-187.

Chano, B.-C., \& Pearson, J. B. 1984 Optimal disturbance reduction in linear multivariable systems IEEE Trans. autom. Control, AC-29, 880-887.

FiedLer, M. 1984 Hankel and Lowner matrices Lin. Alg. and Applic., 58, 75-95.

KaIman, R. E. 1979 On partial realizations, transfer functions, and canonical forms. Acta Polyt. Scand. Ma. 31, 9-32.

Meinguet, J. 1970 On the solubility of the Cauchy interpolation problem. in Proc. Univ. Lancaster Symposium on Approximation theory and application (A. Talbot, ed.) pp. 137-164, Academic Press.

ShapiRo, H. S., \& SHIELDS, A. L. 1961 On some interpolation problems for analytic functions. Amer. J. Math. 83, 513-532.

WALSH, J. 1935 Interpolation and Approximation by Rational Functions in the Complex Domain, New York: AMS Colloquium Publications, Vol. 20.

Youla, D. C., \& SATTO, M. 1967 Interpolation with positive-real functions. J. Franklin Inst. 284, pp. 77-108. 